\title{
Non-Coding RNAs: Master Regulators of Inflammasomes in Inflammatory Diseases
}

\author{
Wei Wang \\ Ni Yang \\ Yu-Hang Yang \\ Ri Wen \\ Chun-Feng Liu \\ Tie-Ning Zhang
}

Department of Pediatrics, Shengjing Hospital of China Medical University, Shenyang, People's Republic of China

Correspondence: Chun-Feng Liu; Tie-Ning Zhang

Department of Pediatrics, Shengjing Hospital of China Medical University, No. 36 SanHao Street, Shenyang City, Liaoning Province, I 10004, People's

Republic of China

$\mathrm{Tel}+86-18940251826$;

+86-18940253339

Email zhliu258@hotmail.com;

cmuztn@vip.qq.com

\begin{abstract}
Emerging data indicates that non-coding RNAs (ncRNAs) represent more than just "junk sequences" of the genome and have been found to be involved in multiple diseases by regulating various biological process, including the activation of inflammasomes. As an important aspect of innate immunity, inflammasomes are large immune multiprotein complexes that tightly regulate the production of pro-inflammatory cytokines and mediate pyroptosis; the activation of the inflammasomes is a vital biological process in inflammatory diseases. Recent studies have emphasized the function of ncRNAs in the fine control of inflammasomes activation either by directly targeting components of the inflammasomes or by controlling the activity of various factors that control the activation of inflammasomes; consequently, ncRNAs may represent potential therapeutic targets for inflammatory diseases. Understanding the precise role of ncRNAs in controlling the activation of inflammasomes will help us to design targeted therapies for multiple inflammatory diseases. In this review, we summarize the regulatory role and therapeutic potential of ncRNAs in the activation of inflammasomes by focusing on a range of inflammatory diseases, including microbial infection, sterile inflammatory diseases, and fibrosis-related diseases. Our goal is to provide new ideas and perspectives for future research.
\end{abstract}

Keywords: ncRNAs, inflammasomes, infection, immune, inflammation, fibrosis

\section{Introduction}

For decades, the miniscule protein-coding portion of the genome was the primary focus of medical research. ${ }^{1}$ However, numerous ncRNAs have been discovered over the past 10 years and have been demonstrated to play important roles in various diseases, including inflammatory diseases. ${ }^{2}$ Normally, inflammation is a defense response of the body to stimuli which is beneficial; however, disorders of inflammation can cause many diseases, including sepsis and autoimmune diseases. ${ }^{3}$ Therefore, orchestrating an effective inflammatory response without harmful immunopathology is an important issue. Exciting research has demonstrated that ncRNAs play a regulatory role in the inflammatory response and are therefore involved in inflammatory diseases. ${ }^{2}$ As an important part of innate immunity, ${ }^{4}$ the activation of the inflammasomes plays a critical role in the regulation of the inflammatory response; imbalance in such regulation can lead to the occurrence of a variety of diseases. Notably, numerous studies emphasize the function of ncRNAs in the fine control of the inflammasomes activation either by directly targeting components of the inflammasomes or by controlling the activity of various regulators of inflammasomes activation, thus representing potential therapeutic targets for inflammatory diseases. ${ }^{5}$ 
The inflammasomes are critical components of the innate immune system. ${ }^{4}$ These differ significantly from membranebound pattern recognition receptors (PRRs). Rather, inflammasomes are multimeric protein complexes that are formed from cytoplasmic PRRs, including members of the evolutionary conserved NOD-like receptors (NLRs), together with absent in melanoma 2 (AIM2)-like receptors (ALRs) and pyrin. These complexes can feature NLRP1, NLRP3, NLRC4, AIM2, and pyrin and can sense microbial pathogens or detect danger associated molecular patterns (DAMPs) and mediate the activation of proinflammatory cytokines as part of the innate immune response. ${ }^{6}$ The assembly and function of inflammasomes involves two different steps: the first initiation step upregulates the expression levels of inflammasomes components while the second activation step induces oligomerization of the inflammasomes complex. ${ }^{7}$ The specific process is shown in Figure 1. The activation of inflammasomes plays a critical role in defense against pathogens and helps to remove damaged and transformed host cells and stimulates an adaptive immune response. Conversely, the aberrant activation of inflammasomes has been linked to some inflammatory diseases.

A firm understanding of the regulation of inflammasomes activation may allow us to induce alterations in this process and improve the prognosis of inflammatory diseases. A substantial number of studies have investigated the regulatory process underlying the activation of inflammasomes from the perspective of ncRNAs in an effort to provide new insights into the regulatory mechanisms associated with inflammatory diseases. ${ }^{5}$ For instance, two studies identified miR-223-3p as the first human miRNA that directly targeted NLRP3. ${ }^{8,9}$ Subsequent studies found that miR-223 could

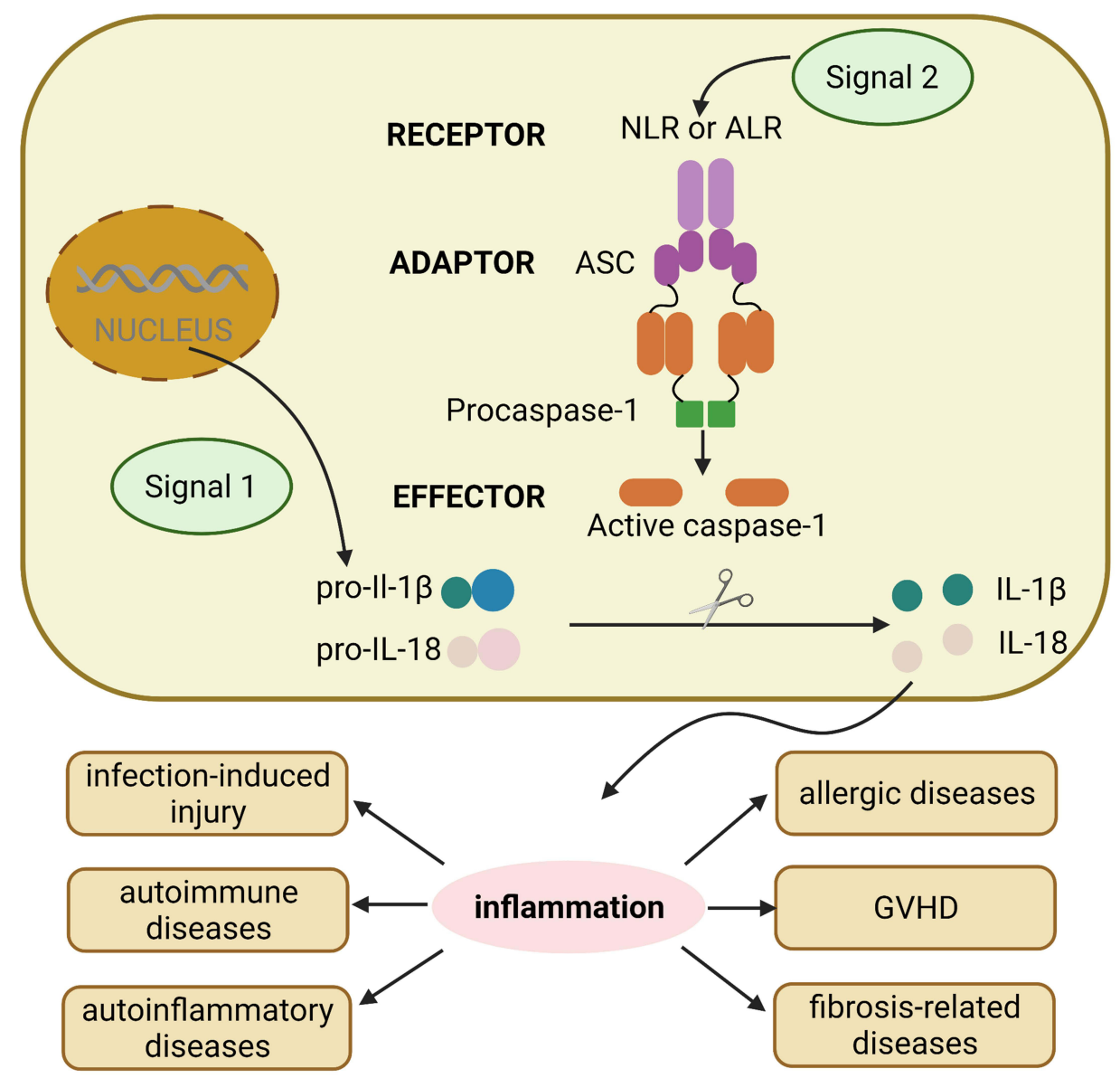

Figure I The general process of activation of different inflammasomes. The assembly and function of inflammasomes involves two different steps: signal I including various PAMPs and DAMPs turns on the first initiation step, which upregulates the expression levels of inflammasomes components such as pro-IL-I $\beta$, pro-IL-I8. Then, signal 2 triggers the second activation step, which induces oligomerization of the inflammasome complex including receptor NLRs and ALRs, adaptor ASC, and effector pro-caspase $-\mathrm{I}$, the assembled pro-caspase-I becomes caspase-I, then cutting pro-IL to activated IL, inducing inflammation response, which plays vital role in inflammation-related diseases including infection-induced injury, autoimmune diseases, autoinflammatory diseases, allergic diseases, GVHD and fibrosis-related diseases.

Abbreviations: PAMPs, pathogen-associated molecular patterns; DAMPs, damage associated molecular patterns; IL, interleukin; NLRs, NOD-like receptors; ALRs, absent in melanoma 2-like receptors; ASC, apoptosis-associated speck-like protein containing caspase recruitment domain; GVHD, graft-versus-host disease. 
participate in different diseases by targeting NLRP3. Long non-coding RNAs (lncRNAs) are non-coding RNAs (ncRNAs) longer than 200 nucleotides. Notably, another study showed that lncRNA could be used as a scaffold to participate in the assembly of inflammasomes. Zhang et al showed that the Neat 1 lncRNA could promote the assembly of inflammasomes, including NLRC4, AIM2, and NLRP3 inflammasomes, thus facilitating the recruitment, maturation, and stabilization of caspase- 1 in activated macrophages. ${ }^{10}$ Furthermore, Neat1 deficiency significantly reduced inflammatory responses by reducing the activation of inflammasomes in mouse models of peritonitis and pneumonia. ${ }^{10}$ Lian et al demonstrated that circHIPK3 could sponge miR-192 and miR-561, and subsequently promote the expression levels of the target genes for miR-192 and miR-561 (Tolllike receptor 4 (TLR4) and NLRP3), thus promoting an inflammatory response; these authors also found that the knockdown of circHIPK3 could suppressed gouty arthritis. ${ }^{11}$ These results showed that ncRNAs played an important regulatory role in the activation of inflammasomes.

Herein, we summarize the role and therapeutic potential of ncRNAs in inflammatory diseases by regulating inflammasomes pathways with a particular emphasis on microbial infection and sterile inflammatory diseases, including microbial infection, infection or sepsis-induced multiple organ failure, autoimmune diseases, autoinflammatory diseases, allergic diseases, graft-versus-host disease (GVHD), and fibrosis related diseases.

\section{Regulatory Mechanisms of ncRNAs}

The ncRNAs can be divided into different classes, broadly based upon their size. Small ncRNAs include miRNAs, transfer RNA (tRNA)-derived small RNAs (tsRNAs), and PIWI-interacting RNAs (piRNAs). At the opposite end of the size spectrum are the lncRNAs; these are defined as untranslated RNAs that are greater than 200 nucleotides (nts) in length, and include a number of subclasses, including pseudogenes and circular RNAs (circRNAs). ${ }^{12}$ At present, the most commonly studied ncRNAs that regulate inflammasomes in the context of inflammatory diseases are miRNAs, lncRNAs, and circRNAs. Therefore, in this review, we focus specifically on these three forms of ncRNAs (Figure 2).

\section{Regulatory Mechanism of miRNAs}

The miRNAs are a form of ncRNAs that are approximately $22 \mathrm{nt}$ in length and constitute almost $1 \%$ of all predicted genes in nematodes, flies and mammals. ${ }^{13}$ Due to their capability to modulate gene expression at the post-transcriptional level either by inhibiting the translation of messenger RNA (mRNA) or by promoting the degradation of mRNA. ${ }^{14}$ The miRNAs have also been shown to be involved in the pathogenesis of many diseases, including neurodegenerative diseases, cardiovascular diseases, and cancer. ${ }^{15-17}$ Mechanistically, miRNAs mediate the post-transcriptional gene silencing of target genes by targeting the $3^{\prime}$ untranslated region of $\mathrm{mRNA}$; the seed region (nucleotides 2-7) at the 5'-end of miRNA is considered to be the crucial sequence. In addition, a single miRNA can target hundreds of mRNAs and influence the expression of many genes that are often involved in a functional interacting pathway. ${ }^{13}$ The miRNA mimics and miRNA inhibitors are currently in preclinical development and have shown promise as novel therapeutic agents. Moreover, several miRNA-targeted therapeutics have reached the clinical development phase, including a mimic of the tumor suppressor miRNA miR-34; this reached Phase I clinical trials for the treatment of cancer. Another example is anti-miRs, which targets miR-122 and reached Phase II trials for the treatment of hepatitis. ${ }^{18}$ An increasing number of studies have demonstrated that miRNAs hold huge potential as diagnostic and prognostic biomarkers, and as predictors of drug response. Regarding the regulation of the inflammasomes pathways by miRNAs, miRNAs could not only directly target the inflammasomes components, such as miR-223 targeting NLRP3, ${ }^{8,9}$ miR-142 targeting apoptosis-associated speck-like protein containing caspase recruitment domain (ASC), ${ }^{19}$ miR-446 targeting prointerleukin $1 \beta$ (pro-IL-1 $\beta$ ), ${ }^{20}$ and miR-379-5p targeting GSDMD, ${ }^{21}$ but also could regulate the upstream pathways of the activation of inflammasomes, such as miR-146a, ${ }^{22}$ miR-96, ${ }^{23}$ and et al regulating NF-kB pathway, miR-33 regulating reactive oxygen species (ROS), ${ }^{24}$ and miR-17, ${ }^{25} \mathrm{miR}-$ $20 \mathrm{a},{ }^{26}$ targeting thioredoxin-interacting protein (TXNIP). ${ }^{27}$ (Figure 3) These miRNAs also play an important role in inflammatory diseases, which will be discussed in detail below.

\section{Regulatory Mechanisms of IncRNAs}

Studies have estimated that the number of lncRNA genes (> $200 \mathrm{nt}$ in length) in humans ranges from less than 20,000 to over $100,000 .^{28}$ Research has shown that IncRNAs play essential roles in the regulation of gene expression despite the fact that they do not code proteins. $^{29}$ Depending on their regulatory patterns, IncRNAs can be further classified into those that act in cis, thus influencing the expression and/or chromatin status 

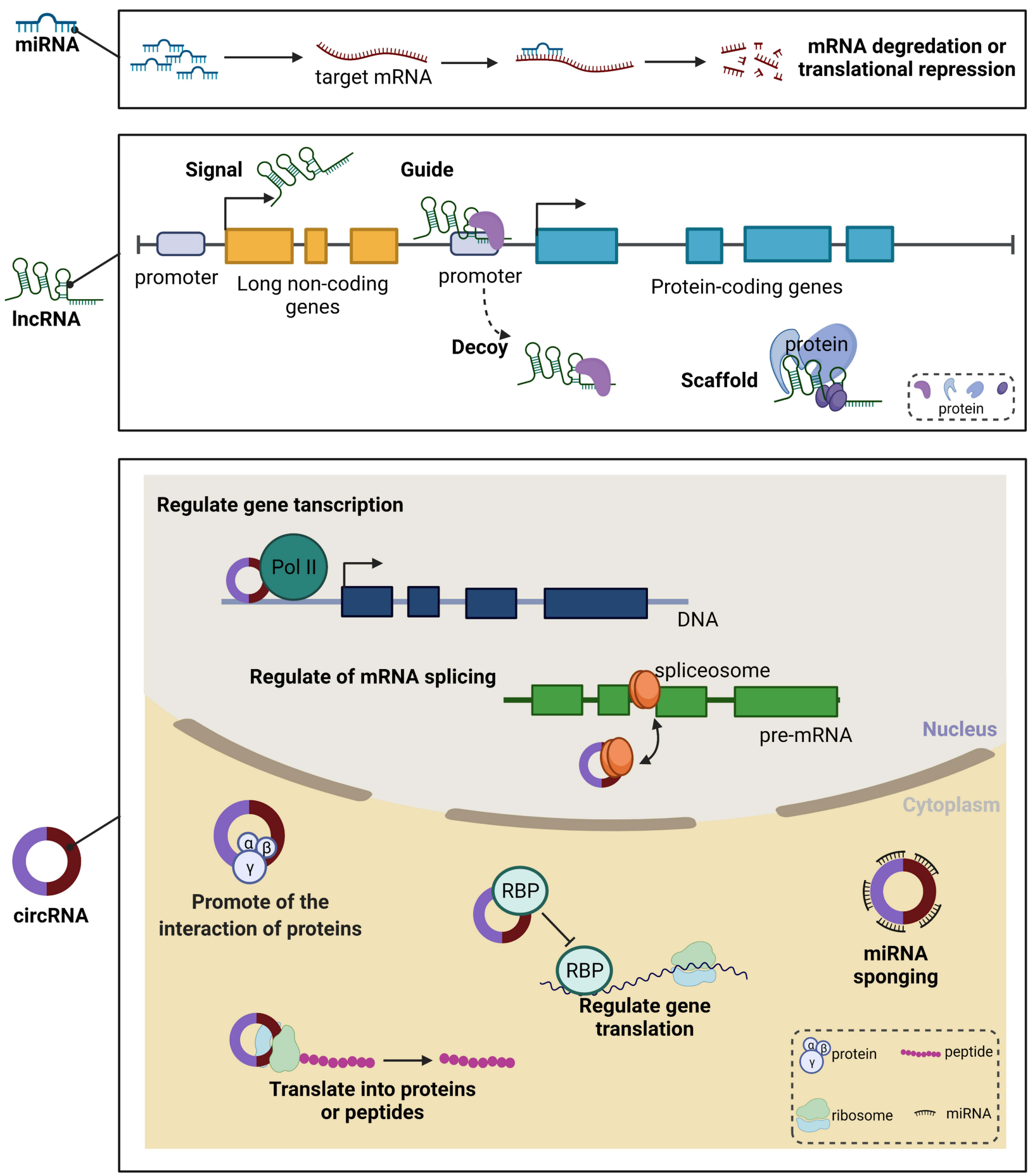

Figure 2 The general regulatory mechanisms of ncRNAs. miRNAs modulate gene expression at the post-transcriptional level either by inhibiting the translation of mRNA or by promoting the degradation of mRNA. IncRNAs can regulate gene expression as signal, guide, decoy and scaffold. circRNAs can exert function at nucleus and cytoplasm. At nucleus, circRNAs can regulate gene transcription and mRNA splicing. At cytoplasm, circRNAs can promote the interaction of proteins, regulate gene translation, be as miRNAs sponging and translate into proteins or peptides.

Abbreviations: mRNA, messenger RNA; miRNAs, micro RNAs; IncRNAs, long non-coding RNAs; circRNAs, circular RNAs; RBP, RNA binding protein.

of nearby genes, and those that execute an array of functions throughout the cell in trans. ${ }^{28}$ According to their genomic position relative to nearby protein-coding genes,
IncRNA can be categorized as intergenic, intronic, bidirectional, sense, antisense, and enhancer lncRNAs. ${ }^{30}$ Notably, antisense transcripts often interact the 


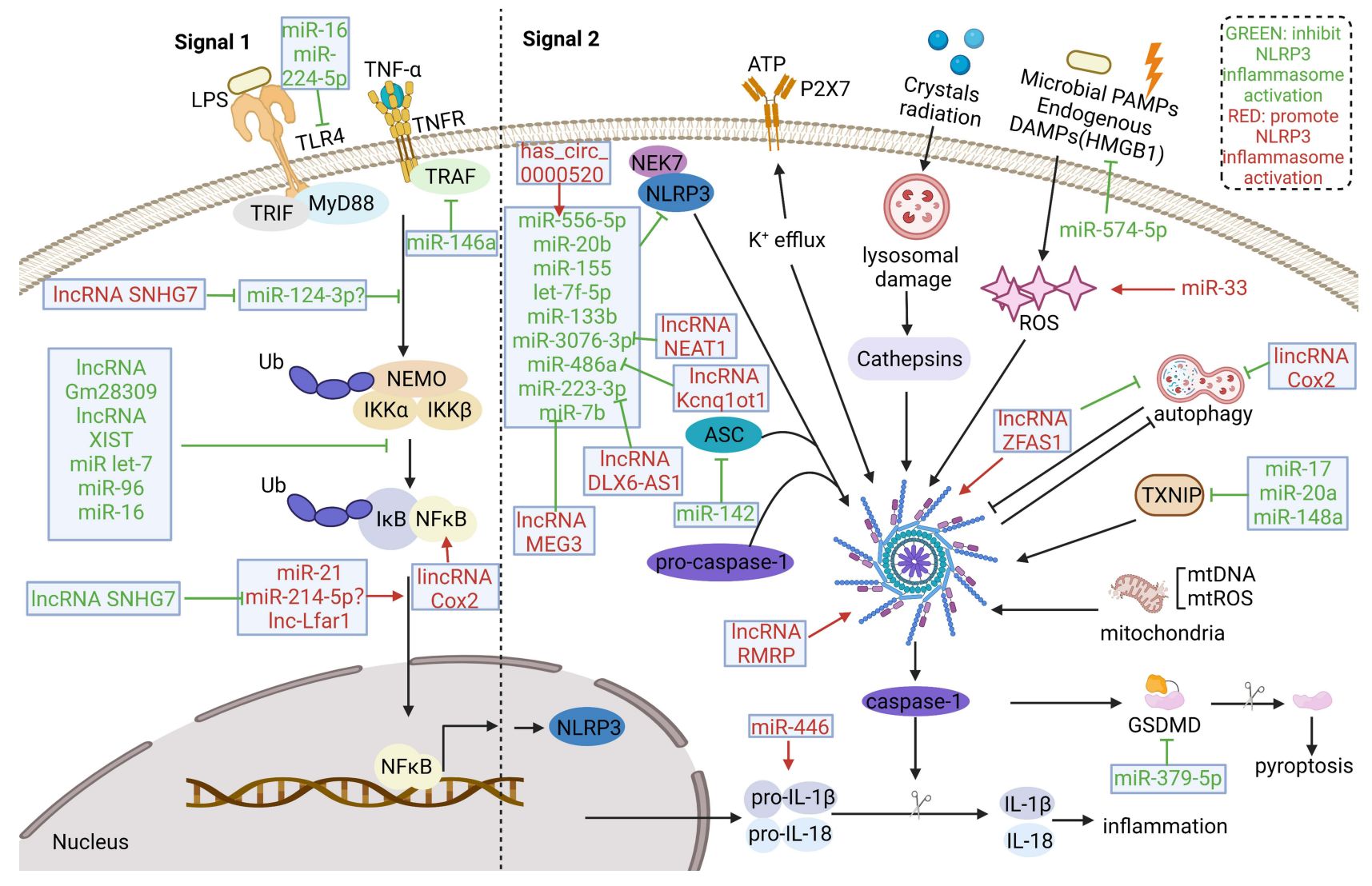

Figure 3 ncRNAs involved in activation of NLRP3 inflammasomes in inflammatory diseases. ncRNAs can regulate both of the two signal for the activation of NLRP3. In the signal I step, multiple molecules or inflammatory cytokines activate NF- $\kappa B$ pathway to up-regulate NLRP3, pro-IL-I $\beta$, pro-IL-I8. Various miRNAs could regulate NF- $\kappa B$ pathway or target components of NF- $\kappa B$ pathway. Several IncRNAs was found to regulate NF- $\kappa B$ pathway as miRNAs' sponge. The signal 2 is motivated by various endogenous and exogenous stimuli, including ATP, crystals radiation, microbial PAMPs and endogenous DAMPs. These stimuli can induce the activation of diverse molecular and cellular signaling pathways, including $\mathrm{K}+$ efflux, lysosomal damage, ROS generation and mitochondrial dysfunction, which are involved in the activation of the NLRP3 inflammasome. In addition, autophagy has interaction with inflammasomes activation. Various miRNAs, several IncRNAs and circRNAs were found to target components or activation signals of NLRP3 inflammasome pathways.

Abbreviations: IL, interleukin; NF- $\mathrm{B}$, nuclear factor-kappa B; ATP, adenosine triphosphatase; PAMPs, pathogen-associated molecular patterns; DAMPs, damage associated molecular patterns; ROS, reactive oxygen species.

corresponding sense transcripts encoded from the opposing gene, thereby interfering with post-transcriptional control by promoting transcript degradation or disrupting translation. Recently, Brocker et al showed that lncRNA Gm15441 could suppress its antisense transcript, encoding thioredoxin interacting protein (TXNIP), thereby inhibiting NLRP3 inflammasome activation. ${ }^{31}$ Interestingly, some lncRNAs have been found to encode small peptides, thus escalating their complexity. ${ }^{32}$ Recently, the study performed by Bhatta et al identified lncRNA 1810058I24Rik, which was downregulated in both human and murine myeloid cells exposed to LPS as well as other Toll-like receptor ligands and inflammatory cytokines and could produce a 47 aa micropeptide, named mitochondrial micropeptide-47 (Mm47), then demonstrated that Mm47-deficient or knockdown cells were compromised for NNLR3 inflammasome responses. ${ }^{33}$ During the transcriptional regulation of target genes, lncRNAs repress or activate genes by functioning as either scaffolds or decoys. ${ }^{34,35}$ Intriguingly, Lin et al demonstrated that lncRNA Platr4 could function to inactivate NIRP3 inflammasome via intercepting NF- $\mathrm{kB}$ signaling. Mechanistically, lncRNA Platr4 could prevent binding of the NF- $\kappa B / R x r \alpha$ complex to the $\kappa B$ sites via a physical interaction, thereby inhibiting the transactivation of NLRP3 and ASC by NF-kB. ${ }^{36}$ In addition, during the activation of inflammasomes, lncRNA Neatl could be used as a scaffold to participate in the assembly of inflammasomes including NLRC4, AIM2, and NLRP3 inflammasome, thus facilitating the recruitment, maturation, and stabilization of caspase-1 in activated macrophages. ${ }^{10}$ LncRNAs can also recruit proteins to chromatin to participate in histone modification and affect gene transcription as guides. For instance, Wang et al uncovered a novel 
IncRNA named Lnc-UC, which could promote expression of Rev-erb $\alpha$ (a known dual NF- $\mathrm{B} / \mathrm{Nlrp} 3$ repressor) to inactive NF- $\kappa \mathrm{B}$ signaling and NLRP3 inflammasome in macrophages. In the molecular level, Lnc-UC could interact with chromobox 1 to reduce $\mathrm{H} 3 \mathrm{~K} 9 \mathrm{me} 3$ at Rev-erb $\alpha$ promoter and to induce Rev-erba transcription and expression. ${ }^{37}$ Conversely, Cai et al showed that lncRNA MALAT1 could promote neuroinflammation by recruiting enhancer of zeste homologue 2 to the promoter of nuclear factor erythroid 2-related factor 2 (Nrf2), suppressing Nrf2 expression epigenetically, thereby inducing inflammasomes activation in parkinson's diseases mouse and microglial cell models. ${ }^{38}$ LncRNAs can also regulate gene transcription as enhancers. ${ }^{39-41}$ They can also cotranscriptionally regulate mRNA transcripts by altering their splicing patterns. ${ }^{42}$ At the post-transcriptional level, lncRNAs function as miRNAs sponges, which can indirectly represses the expression of an mRNA that would be targeted by miRNAs. ${ }^{43}$ Several studies have showed that lncRNAs could regulate the expression of components of inflammasome-related pathways via miRNAs sponges. For instance, Chi et al demonstrated that IncRNA HOTAIR could promote renal inflammatory response in hyperuricemia mice by competitively binding miR-22 to regulate NLRP3 expression. ${ }^{44}$ In addition, the study conducted by Zhou et al showed that overexpression of IncRNA TUG1 could promote NLRP3 inflammasome-mediated cardiomyocyte pyroptosis through targeting miR-186-5p/ $\mathrm{X}$-Linked inhibitor of apoptosis axis in coronary microembolization-induced myocardial damage. ${ }^{45}$ Moreover, lncRNAs can affect the stability of mRNA transcripts, ${ }^{46}$ and modulate mRNA translation by binding to ribosomes or mRNA transcripts during translation. ${ }^{47}$ Furthermore, lncRNA could regulate protein nucleoplasmic shuttle. For example, lincRNA-Cox 2 could bind NF-kB p65 and promote its nuclear translocation and transcription, modulating the expression of inflammasome sensor NLRP3 and adaptor ASC, thereby enhancing the activation of NLRP3 inflammasome. ${ }^{48}$ Thus, evidence has demonstrated that IncRNAs can participate in the regulation of gene expression via a variety of mechanisms and increasing number lncRNAs have been found to exert function in the activation of inflammasomes.

\section{Regulatory Mechanisms of circRNAs}

CircRNAs consisting of a circular configuration through a typical $5^{\prime}$ to $3^{\prime}$-phosphodiester bond, has been recently recognized as a new class of functional molecules. The
circRNA has no $5^{\prime}$ or $3^{\prime}$ free terminus and is much more stable in cellular environment. RNA molecules with a circular configuration were first discovered four decades ago. Over recent years, circRNAs have been found to be involved in the regulation of many diseases, including cancer, cardiovascular diseases, and metabolic diseases. ${ }^{49,50}$ However, the function of circRNAs remains largely unknown. A small number of circRNAs have been shown to be involved in post-transcriptional regulation by functioning as "sponges" of miRNAs, thus reducing their ability to target mRNAs. ${ }^{49,50}$ At present, the main focus on the role of circRNAs in the regulation of inflammasomes is through sponging miRNAs. For instance, Lian et al showed that circHIPK3 could sponge miR-192 and miR561 to promote TLR4 and NLRP3 expressions, thereby promoting inflammation in gouty arthritis. ${ }^{11}$ Similarly, the study performed by Bian et al demonstrated that circHelz could activate NLRP3 inflammasome to promote myocardial injury by sponging miR-133a-3p in mouse ischemic heart. $^{51}$ In addition, exosomes are microvesicles that can contain ncRNAs such as miRNAs and circRNAs, and are secreted by many different types of cells. Stem cellderived exosomes can be used as carriers to treat diseases. Recently, Yan et al reported that human umbilical cord mesenchymal stem cells- derived exosomes could prevent hindlimb ischemic injury by releasing cicrHIPK3, which could in turn down regulate miR-421, resulting in increased expression of FOXA3a, leading to inhibition of NLRP3 inflammasome mediated pyroptosis. ${ }^{52}$ Intriguingly, there is an interaction between autophagy and inflammasomes activation. Zhang et al showed that circARF3 could alleviate mitophagy-mediated inflammation induced by NLRP3 inflammasome activation via targeting miR-103/TRAF3 in mouse adipose tissue. ${ }^{53}$ Except for sponging miRNAs, circRNAs have been found to be involved in transcriptional activation by recruiting transcription factors. For example, Yang et al suggested that circ-HuR suppressed the growth, invasion, and metastasis, of gastric cancer cells in vitro and in vivo. Mechanistically, circ-HuR interacted with CCHC-type zinc finger nucleic acid binding protein, and subsequently restrained its binding to the HuR promoter, thus resulting in the downregulation of $\mathrm{HuR}$ and the repression of tumor progression. ${ }^{54}$ Moreover, several studies have shown that circRNAs sponged splicing factors to regulate gene splicing. For example, Barbagallo et al found that circSMARCA5 represented a promising and druggable tumor suppressor in glioblastoma multiforme and 
suggested that circSMARCA5 might exert function by tethering RNA binding protein serine and arginine rich splicing factor 1 , a splicing factor known to be a positive controller of cell migration. ${ }^{55}$ Furthermore, circRNAs can also regulate target gene translation, ${ }^{56}$ or act as an adaptor to modulate protein-protein interactions and their activities. ${ }^{57}$ In addition, an increasing body of evidence indicates that circRNAs may not be a real class of ncRNAs because some are translatable. ${ }^{58}$ These mechanisms may be the potential mechanisms for circRNAs to regulate inflammasomes activation. However, circRNAs are known to play important functions in gene transcription and post-transcription regulation and deserve our further attention, especially in inflammasomes activation.

\section{The Roles of ncRNAs in Inflammasomes Activation in Inflammatory Diseases}

\section{The Roles of ncRNAs in Inflammasomes Activation in Infectious Diseases}

Inflammation is the typical response of the host immune system to microbial infection. However, excessive and unresolved inflammation could contribute to tissue injury such as acute lung injury (ALI), acute liver failure (ALF), or even the occurrence of sepsis. ${ }^{59}$ Notably, sepsis refers to a lifethreatening organ dysfunction caused by a dysregulated host response to infection that is characterized by systemic inflammatory response syndrome. Sepsis could also lead to ALI, ALF, acute kidney injury (AKI), and heart dysfunction. ${ }^{60}$ The incidence of sepsis is as high as $1-2 \%$ in all hospitalized patients and therefore poses a serious threat to human life. Due to a lack of specific drugs, sepsis can only be treated by antibiotic treatment, supportive treatment and symptomatic treatment. ${ }^{61}$ Therefore, it is vital to gain a deeper understanding of microbial infection, infectioninduced tissue damage, and sepsis-induced multiple organ dysfunction so that we can identify new therapeutic targets. Recent studies have found that the excessive activation of inflammasomes contributes to microbial infection-induced inflammation, ${ }^{43}$ infection-induced tissue injury, ${ }^{62-64}$ and sepsis-induced AKI, ${ }^{65}$ and heart dysfunction. ${ }^{66}$ Moreover, an increasing number of studies has shown that the use of ncRNAs to target inflammasome pathways or the molecules upstream of inflammasome pathways could alleviate microbial infection-induced injury and sepsis-induced multiple organ dysfunction, thus facilitating the future development of therapeutic strategies (Table 1).
The Roles of ncRNAs in Regulating Inflammasomes Activation in Different Types of Microbial Infection

Many studies have shown that miRNAs can directly target the key components of the inflammasome pathways involved in microbial infection, particularly NLRP3. The NLRP3 inflammasome is the most widely studied inflammasome and is known to be activated in many inflammatory diseases, including microbial infection. The activation process of NLRP3 inflammasome is showed in Figure 3. Several miRNAs have been found to be significantly reduced in patient serum with the potential as biomarkers, and could reduce inflammation by directly targeting NLRP3. For example, Lou et al showed that miR-20b was downregulated and NLRP3 was upregulated in the macrophages of both patients and mice with tuberculosis (TB). ${ }^{67}$ Treatment with a miR-20b mimic alleviated the inflammatory response and deactivated the NLRP3/caspase-1/IL-1 $\beta$ pathway in a mouse model of TB and in alveolar epithelial cells co-cultured with macrophages; these effects occurred via the direct targeting of NLRP3. ${ }^{67}$ Moreover, a study involving Treponema pallidum subspecies pallidum ( $\mathrm{T}$ pallidum) infection showed that a dramatic reduction in the serum levels of miR-223-3p of patients with syphilis compared with healthy controls, which could target NLRP3 to suppress inflammasome activation and pyroptosis in T pallidum-induced Human Umbilical Vein Endothelial Cells (HUVECs), thus indicating a potential target for syphilis-induced endothelial injury. ${ }^{68}$

Apart from targeting NLRP3 directly, ncRNAs can also regulate the NLRP3 inflammasome activation process. The NLRP3 inflammasome can be activated by a diverse range of stimuli and a 2-signal model has been proposed for to explain this process (Figure 3). Signal 1 is provided by microbial molecules or endogenous cytokines, in which the activation of nuclear factor kappa-light-chain-enhancer of activated B cells (NF- $\kappa \mathrm{B}$ ) leads to the up-regulation of NLRP3 and pro-IL-1 $\beta{ }^{69,70}$ For instance, Xue et al showed that miR-21 was a critical positive regulator of the NF- $\kappa \mathrm{B}$ pathway and NLRP3 inflammasome via A20 in myeloid cells from both humans and mice. The knockout of miR-21 was shown to reduce mortality rates in a lipopolysaccharide (LPS)-induced mouse model of septic shock. ${ }^{71}$ Also, bioinformatic analysis showed that miR-21 levels in peripheral blood from patients with septic shock were elevated. $^{71}$ In addition, a study conducted by Lee et al demonstrated that miRNA-rich extracellular vesicles (EVs) could contribute to innate immune responses after bacterial lung infection. Mechanistically, miRNAs 
Table I The Effect of ncRNAs in Activation of Inflammasomes in Different Inflammatory Diseases

\begin{tabular}{|c|c|c|c|c|c|}
\hline ncRNA & Model & Target & Function in Inflammasome Activation & Level & Reference \\
\hline \multicolumn{6}{|c|}{ Microbial infection } \\
\hline miR-20b & $\begin{array}{l}\text { Macrophages of patients and mice with TB / } \\
\text { alveolar epithelial cells co-cultured with } \\
\text { macrophages }\end{array}$ & NLRP3 & $\begin{array}{l}\text { Reduce inflammatory response by direct } \\
\text { target NLRP3 }\end{array}$ & Down & [67] \\
\hline miR-223-3p & $\begin{array}{c}\text { Patients with syphilis/ T pallidum-induced } \\
\text { HUVECs }\end{array}$ & NLRP3 & $\begin{array}{l}\text { Suppress inflammasome activation and } \\
\text { pyroptosis by direct target NLRP3 }\end{array}$ & Down & [68] \\
\hline miR-2I & $\begin{array}{l}\text { LPS-induced mice model of septic shock/LPS- } \\
\text { induced myeloid cells }\end{array}$ & $\mathrm{A} 20$ & $\begin{array}{l}\text { Promote NLPR3 inflammasome activation by } \\
\qquad A 20 / N F-\kappa B \text { pathway }\end{array}$ & Up & [7I] \\
\hline $\begin{array}{l}\text { miRs contained in } \\
\text { alveolar epithelial } \\
\text { type-I cells-EVs }\end{array}$ & $\begin{array}{l}\text { P. aeruginosa-induced alveolar macrophages } \\
\text { and mice }\end{array}$ & - & $\begin{array}{c}\text { Promote NLRP3 inflammasome activation, } \\
\text { neutrophil recruitment, and MI-macrophage } \\
\text { polarization }\end{array}$ & Up & [72] \\
\hline miR-223 & $\begin{array}{l}\text { LPS-induced bovine endometritis and bovine } \\
\text { endometrial epithelial cell (BEND) line }\end{array}$ & NLRP3 & $\begin{array}{l}\text { Reduce inflammatory response by direct } \\
\text { target NLRP3 }\end{array}$ & Up & [73] \\
\hline miR-223-3p & H. pylori-induced THP-I monocytes & NLRP3 & $\begin{array}{c}\text { Reduce NLRP3 inflammasome activation and } \\
\text { increase } \mathrm{H} \text {. pylori survival }\end{array}$ & Up & [74] \\
\hline miR-223 & LPS-induced human dental pulp fibroblasts & NLRP3 & $\begin{array}{l}\text { Reduce inflammatory response by direct } \\
\text { target NLRP3 }\end{array}$ & Down & [75] \\
\hline miR-223 & influenza $A$ virus (IAV)-induced lung epithelial & NLRP3 & $\begin{array}{l}\text { Reduce inflammatory response by direct } \\
\text { target NLRP3 }\end{array}$ & Down & [76] \\
\hline miR-I55 & $\begin{array}{l}\text { Porphyromonas gingivalis-induced } \\
\text { macrophages }\end{array}$ & NLRP3 & $\begin{array}{c}\text { Reduce NLRP3 inflammasome-mediated } \\
\text { pyroptosis }\end{array}$ & Up & [77] \\
\hline $\begin{array}{l}\text { IncRNA } \\
\text { Gm28309 }\end{array}$ & Brucella strain-induced RAW264.7 cells & кB-Ras2 & $\begin{array}{l}\text { Reduce inflammation response by miR-3068- } \\
\qquad 5 \mathrm{p} / \mathrm{NF}-\mathrm{KB} / \mathrm{NLRP3}\end{array}$ & Down & [78] \\
\hline IncRNA XIST & $\begin{array}{l}\text { E. coli or } S \text {. aureus-induced mammary } \\
\text { alveolar cell-T (MAC-T) cells }\end{array}$ & NF- $\kappa B$ & $\begin{array}{l}\text { Reduce inflammatory response by NF-KB/ } \\
\text { NLRP3 pathway }\end{array}$ & Up & [79] \\
\hline \multicolumn{6}{|c|}{ ALI } \\
\hline $\mathrm{miR}-223 / \mathrm{l} 42 \mathrm{EVs}$ & $\begin{array}{l}\text { BALF and serum of lung infection-associated } \\
\qquad \text { ALI in patients/ LPS or } \\
\text { Klebsiella pneumoniae-induced macrophages }\end{array}$ & $\begin{array}{l}\text { NLRP3/ } \\
\text { ASC }\end{array}$ & $\begin{array}{l}\text { Reduce inflammatory response by direct } \\
\text { target NLRP3 and ASC }\end{array}$ & Up & [19] \\
\hline miR-223 & MTDs-induced mice /ARDS patients & NLRP3 & $\begin{array}{l}\text { Limit the number of Ly6G+ neutrophils and } \\
\text { inhibit the activity of the NLRP3 } \\
\text { inflammasome }\end{array}$ & Up & [82] \\
\hline IncMEG3 & $\begin{array}{l}\text { LPS-induced mice and mouse alveolar } \\
\text { macrophage NR8383 }\end{array}$ & miR-7b & $\begin{array}{l}\text { Promote LPS-induced ALI by acting as } \\
\text { a molecular sponge for miR-7b to modulate } \\
\text { NLRP3 directly }\end{array}$ & Up & [83] \\
\hline miR-16 & LPS-induced ALI mice and A549 cells & TLR4 & $\begin{array}{l}\text { Reduce inflammatory response by TLR4/NF- } \\
\qquad \mathrm{KB} / \mathrm{NLRP3} \text { pathway }\end{array}$ & Down & [85] \\
\hline miRNA-466 & $\begin{array}{l}\text { EVs isolated from the BALF of LPS-induced } \\
\text { ARDS mice/LPS-induced macrophages }\end{array}$ & Pro-ILI $\beta$ & $\begin{array}{l}\text { Promote inflammation by up-regulating pro-IL } \\
\qquad-1 \beta\end{array}$ & Up & [20] \\
\hline miR-574-5p & $\begin{array}{l}\text { LPS-induced human pulmonary microvascular } \\
\text { endothelial cells (HPMECs) and ARDS mice }\end{array}$ & HMGBI & $\begin{array}{l}\text { Suppress NF-kB signaling pathway and NLRP3 } \\
\text { inflammasome activation by targeting HMGBI }\end{array}$ & Up & [88] \\
\hline
\end{tabular}


Table I (Continued).

\begin{tabular}{|c|c|c|c|c|c|}
\hline ncRNA & Model & Target & Function in Inflammasome Activation & Level & Reference \\
\hline miR-223 & LPS-induced A549 cells & NLRP3 & $\begin{array}{c}\text { Suppress NLRP3 inflammasome by targeting } \\
\text { NLRP3 directly and the TLR4/NF-KB signaling } \\
\text { pathway via RHOB }\end{array}$ & Down & [89] \\
\hline miR-199a-3p & $\begin{array}{l}\text { LPS-induced A549 and the human } \\
\text { macrophage cell line U937 }\end{array}$ & NLRPI & $\begin{array}{l}\text { Reduce inflammation by targeting NLRPI } \\
\text { directly }\end{array}$ & Down & [90] \\
\hline \multicolumn{6}{|c|}{ ALF } \\
\hline miR-223-3p & LPS/GaIN-induced ALF mice & NLRP3 & $\begin{array}{l}\text { Reduce LPS/D-GalN-induced liver injury by } \\
\text { targeting NLRP3 directly }\end{array}$ & - & [93] \\
\hline miR-I7 exosomes & LPS/GaIN-induced ALF mice and LPS-induced & TXNIP & $\begin{array}{l}\text { Alleviate LPS/GalN-induced ALF by reducing } \\
\text { TXNIP/NLRP3 inflammasome activation }\end{array}$ & - & [25] \\
\hline \multicolumn{6}{|c|}{ Sepsis-induced heart dysfuncti-on } \\
\hline IncRNA ZFASI & $\begin{array}{l}\text { CLP-induced sepsis mice and LPS-induced } \\
\text { primary cardiomyocytes }\end{array}$ & $\begin{array}{l}\text { miR-590- } \\
3 p\end{array}$ & $\begin{array}{l}\text { Promote LPS-induced NLRP3 mediated } \\
\text { Pyroptosis and inhibit autophagy by AMPK/ } \\
\text { mTOR pathway though sponging miR-590-3p }\end{array}$ & Up & [96] \\
\hline \multicolumn{6}{|c|}{ AKI } \\
\hline $\begin{array}{l}\text { IncRNA DLX6- } \\
\text { ASI }\end{array}$ & $\begin{array}{l}\text { Septic AKI patients and LPS-induced HK-2 } \\
\text { cells }\end{array}$ & $\begin{array}{l}\operatorname{miR}-223- \\
3 p\end{array}$ & $\begin{array}{l}\text { Promote LPS-mediated cytotoxicity and } \\
\text { pyroptosis by miR-223-3p/NLRP3 pathway }\end{array}$ & Up & [97] \\
\hline IncRNA RMRP & AKI patients and LPS-induced HK-2 cells & - & $\begin{array}{l}\text { Promote inflammation by activating the } \\
\text { NLRP3 inflammasome }\end{array}$ & Up & [98] \\
\hline $\begin{array}{l}\text { IncRNA } \\
\text { Kenqlot I }\end{array}$ & sC5b-9-induced podocytes & $\begin{array}{l}\text { miR-486a- } \\
\quad 3 p\end{array}$ & $\begin{array}{l}\text { Promote podocyte pyroptosis by sponging } \\
\text { miR-486a-3p/NLRP3 }\end{array}$ & Up & [99] \\
\hline miR-223-3p & LPS-induced HK-2 cells & NLRP3 & $\begin{array}{l}\text { Inhibit NLRP3 inflammasome activation by } \\
\text { targeting NLRP3 directly }\end{array}$ & - & {$[100]$} \\
\hline \multicolumn{6}{|c|}{ Auto-immune diseases } \\
\hline miR-223 & IBD patients/ miR-223-/y mice & NLRP3 & $\begin{array}{l}\text { Reduce myeloid-driven experimental colitis by } \\
\text { targeting NLRP3 directly }\end{array}$ & Up & {$[108]$} \\
\hline miR-223-3p & EAM mice & NLRP3 & $\begin{array}{l}\text { Promote polarization of dendritic cells (DCs) } \\
\text { towards a tolerogenic DC phenotype and } \\
\text { regulatory T cell (Treg) generation by } \\
\text { inhibiting NLRP3 inflammasome activation }\end{array}$ & Down & [109] \\
\hline let-7f-5p & $\begin{array}{l}\text { Bone marrow-derived mesenchymal stem } \\
\text { cells from systemic lupus erythematosus } \\
\text { patients }\end{array}$ & NLRP3 & $\begin{array}{l}\text { Attenuate inflammation by targeting NLRP3 } \\
\text { directly }\end{array}$ & Up & {$[110]$} \\
\hline miR-I46a & $\begin{array}{l}\text { Monosodium urate (MSU)-induced gouty } \\
\text { arthritis mice }\end{array}$ & $\begin{array}{l}\text { TRAF6/ } \\
\text { IRAKI }\end{array}$ & $\begin{array}{l}\text { Reduce paw swelling and index and ankle joint } \\
\text { swelling by targeting TRAF6 and IRAKI } \\
\text { mediated NLRP3 inflammasome activation }\end{array}$ & - & [22] \\
\hline miR-I55 & $\begin{array}{l}\text { Imiquimod-induced psoriasis mice / } \\
\text { keratinocyte-induced } \mathrm{HaCaT} \text { cells }\end{array}$ & NLRP3 & $\begin{array}{l}\text { Promote psoriasis-induced inflammation by } \\
\qquad \text { NLRP3/caspase-I signaling }\end{array}$ & Up & {$[111]$} \\
\hline miR-33 & RA patients/ primary macrophages & - & $\begin{array}{l}\text { Promote NLRP3 inflammasome activation by } \\
\text { increasing accumulation of cellular ROS }\end{array}$ & Up & [24] \\
\hline miR-20a & $\begin{array}{l}\text { Primary fibroblast-like synoviocytes (FLSs) } \\
\text { isolated from adjuvant-induced arthritis (AA) }\end{array}$ & TXNIP & $\begin{array}{l}\text { Reduce formation of the NLRP3 } \\
\text { inflammasome by targeting TXNIP directly }\end{array}$ & - & [26] \\
\hline
\end{tabular}


Table I (Continued).

\begin{tabular}{|c|c|c|c|c|c|}
\hline ncRNA & Model & Target & Function in Inflammasome Activation & Level & Reference \\
\hline lincRNA-Cox2 & $\begin{array}{l}\text { LPS-induced microglial cells, BMDMs and } \\
\text { BV2/ autoimmune encephalomyelitis mice }\end{array}$ & NF- $\kappa B$ & $\begin{array}{l}\text { Promote NLRP3 inflammasome activation and } \\
\text { inhibit autophagy by bonding to NF-kB p65 } \\
\text { directly and promote p } 65 \text { transfer to nucleus }\end{array}$ & - & [48] \\
\hline \multicolumn{6}{|c|}{ Auto-inflammatory diseases } \\
\hline miR-197-3p & FMF patients & ILIRI & $\begin{array}{l}\text { Inhibit NLRP3 inflammsome activation by } \\
\text { targeting ILIRI }\end{array}$ & Down & [122] \\
\hline \multicolumn{6}{|c|}{ Allergic diseases } \\
\hline has_circ_0000520 & AR patients and mice & $\begin{array}{l}\text { miR-556- } \\
5 p\end{array}$ & $\begin{array}{c}\text { Promote NLRP3-mediated cell pyroptosis by } \\
\text { direct targeting NLRP3 }\end{array}$ & Up & [136] \\
\hline miR-224-5p & AR mice & TLR4 & $\begin{array}{c}\text { Inhibit NLRP3 inflammasomes activation by } \\
\text { direct targeting TLR4 }\end{array}$ & Down & [137] \\
\hline miR-133b & AR mice & NLRP3 & $\begin{array}{l}\text { Inhibit NLRP3 inflammasomes activation by } \\
\text { direct targeting NLRP3 }\end{array}$ & Down & [138] \\
\hline \multicolumn{6}{|c|}{ GVHD } \\
\hline IncRNA NEATI & $\begin{array}{l}\text { EAM and heart transplantation mice/ } \\
\text { tolerogenic dendritic cells }\end{array}$ & $\begin{array}{l}\operatorname{miR}-3076- \\
3 p\end{array}$ & $\begin{array}{l}\text { Promote immune tolerance by targeting } \\
\qquad \text { NLRP3 }\end{array}$ & - & [140] \\
\hline miR-I55 & $\begin{array}{l}\text { LPS-stimulated DCs/ allogeneic } \\
\text { hematopoietic cell transplantation recipient } \\
\text { mice }\end{array}$ & - & $\begin{array}{l}\text { Promote GVDH by increasing NLRP3 } \\
\text { inflammasome }\end{array}$ & Up & [143] \\
\hline \multicolumn{6}{|c|}{ Other sterile inflammatory diseases } \\
\hline miR-233 & $\begin{array}{l}\text { Anterior cruciate ligament transection- } \\
\text { induced osteoarthritis (OA) rat }\end{array}$ & NLRP3 & $\begin{array}{l}\text { Inhibit NLRP3 inflammasome activation by } \\
\text { targeting NLRP3 directly }\end{array}$ & Up & [144] \\
\hline IncRNA SNHG7 & OA rat/ IL-I $\beta$-induced chondrocytes & FSTL-I & $\begin{array}{c}\text { Promote FSTL-I-mediated NLRP3 } \\
\text { inflammasome activation and TLR4/NF- } \mathrm{kB} \\
\text { signaling pathways by targeting miR-I24-3p }\end{array}$ & Up & [146] \\
\hline IncRNA SNHG7 & OA patients/ IL-I $\beta$-induced chondrocytes & PPARGCIB & $\begin{array}{c}\text { Inhibiting NLRP3 inflammasome activation } \\
\text { and apoptosis via the miR-2 I4-5p/PPARGCIB } \\
\text { axis }\end{array}$ & Down & [147] \\
\hline miR-I48a & $\begin{array}{l}\text { Alcoholic hepatitis (AH) patients/ } \mathrm{AH} \text { and } \\
\text { alcoholic liver disease mice/ alcohol-induced } \\
\text { hepatocyte }\end{array}$ & TXNIP & $\begin{array}{c}\text { Ameliorate alcohol-induced inflammasome } \\
\text { activation and pyroptosis in hepatocytes } \\
\text { through TXNIP inhibition }\end{array}$ & Down & [27] \\
\hline \multicolumn{6}{|c|}{ Fibrosis related diseases } \\
\hline miR-2I & $\begin{array}{l}\text { Liver fibrosis patient/ BDL or Angll infusion- } \\
\text { induced liver fibrosis rat/ Angll-induced } \\
\text { hepatic stellate cells (HSCs) }\end{array}$ & $\begin{array}{l}\text { Spryl/ } \\
\text { Smad7 }\end{array}$ & $\begin{array}{c}\text { Promote NLRP3 inflammasomes activation } \\
\text { and resultant collagen production via the } \\
\text { Spryl/ERK/NF-kB and Smad7/Smad2/3/NOX } 4 \\
\text { pathways by targeting Spryl and Smad7 } \\
\text { directly }\end{array}$ & Up & {$[151]$} \\
\hline miR-379-5p & $\begin{array}{l}\text { Arsenite-exposed mice/arsenite-induced } \\
\text { HSCs and human hepatic cells }\end{array}$ & GSDMD & $\begin{array}{l}\text { Inhibit arsenite-induced increase in GSDMD } \\
\text { levels and the release of IL-I } \beta \text { by targeting } \\
\text { GSDMD directly }\end{array}$ & Down & {$[21]$} \\
\hline
\end{tabular}

(Continued) 
Table I (Continued).

\begin{tabular}{|c|c|c|c|c|c|}
\hline ncRNA & Model & Target & Function in Inflammasome Activation & Level & Reference \\
\hline Inc-Lfarl & $\begin{array}{l}\text { CCl4-and bile duct ligation (BDL) induced } \\
\text { liver fibrosis mice/LPS or IFN- } \gamma \text {-induced } \\
\text { primary KCs, RAW264.7 cells and bone } \\
\text { marrow-derived macrophages (BMMs) }\end{array}$ & $N F-\kappa B$ & $\begin{array}{l}\text { Promote carbon tetrachloride (CCl4)- and } \\
\text { BDL-induced proinflammatory MI } \\
\text { macrophage activation and NLRP3 } \\
\text { inflammasome-mediated pyroptosis by NF-KB } \\
\text { pathway }\end{array}$ & Down & {$[152]$} \\
\hline miR-2I & BLM-induced lung fibrosis rat & Spryl & $\begin{array}{l}\text { Inhibit Angll-induced activation of the NLRP3 } \\
\text { inflammasome by targeting Spryl in lung } \\
\text { fibroblasts }\end{array}$ & Up & [157] \\
\hline $\begin{array}{l}\text { MenSCs-Exo } \\
\text { containing miR } \\
\text { let-7 }\end{array}$ & BLM-induced lung fibrosis mice & LOXI & $\begin{array}{l}\text { Inhibit activation of NLRP3 inflammasome by } \\
\text { affecting ROS and mtDNA damage via } \\
\text { targeting LOXI directly }\end{array}$ & - & [159] \\
\hline miR-96 & $\begin{array}{l}\text { Carbon black nanoparticle-induced lung } \\
\text { fibrosis rat and human bronchial epithelial } \\
\text { cells }\end{array}$ & FOXO3a & $\begin{array}{l}\text { Attenuate fibrosis through inhibiting NLRP3 } \\
\text { inflammasome by targeting FOXO3a }\end{array}$ & Up & [23] \\
\hline
\end{tabular}

Abbreviations: ACLT, anterior cruciate ligament transection; AH, alcoholic hepatitis; AKI, acute kidney injury; ALF, acute liver failure; ALI, acute lung injury; ARDS, acute respiratory distress syndrome; ASC, apoptosis-associated speck-like protein containing CARD; BALF, bronchoalveolar lavage fluid; BDL, bile duct ligation; BEND cell, bovine endometrial epithelial cell; BLM, Bleomycin; BMMs, bone marrow-derived macrophages; CCl4, carbon tetrachloride; CLP, cecal ligation and puncture; DAMPs, damageassociated molecular patterns; DCs, dendritic cells; E. coli, Escherichia coli; EAM, experimental autoimmune myocarditis; EVs, extracellular vesicles; FSTL-I, Follistatin-like Protein I; GVDH, graft-versus-host disease; H. pylori, Helicobacter pylori; HMGBI, high-mobility group protein BI; HSCs, hepatic stellate cells; HUVECs, Human Umbilical Vein Endothelial Cells; IAV, influenza A virus; IBD, linflammatory bowel disease; IL-I $\beta$, Interleukin-I beta; IRAKI, interleukin-I receptor-associated kinase; LPS, lipopolysaccharide; MAC-T cell, mammary alveolar cell-T; MSU, monosodium urate; MTDs, mitochondrial DAMPs; NLRPI, Nucleotide- binding oligomerization domain, leucinerich repeat and pyrin domain- containing I; NLRP3, Nucleotide- binding oligomerization domain, leucine- rich repeat and pyrin domain- containing 3; OA, osteoarthritis; P. aeruginosa, Pseudomonas aeruginosa; RA, rheumatoid arthritis; ROS, reactive oxygen species; S. aureus, Staphylococcus aureus; TB, tubercle bacillus; TLR4, Toll-like receptor 4; TRAF6, TNF receptor associated factor 6; Treg, regulatory T cell; TXNIP, thioredoxin-interacting protein.

contained in alveolar epithelial type-I cells (ATIs)-EVs including miR-17, miR-93, miR-221, miR-320, miR-92a, mir-199a and mir-330, were actively delivered into alveolar macrophages, subsequently promoting NLRP3 inflammasome activation, neutrophil recruitment, and M1macrophage polarization in response to $P$. aeruginosa pneumonia in vitro and in vivo. ${ }^{72}$ These studies involving miRNAs provide a novel insight into the development of biomarkers, therapeutic strategies, and the mechanisms that underlie microbial infection.

Furthermore, miR-223 and miR-155 were found to be involved in infection by targeting NLRP3 in both animal and cell models. Zhao et al reported that miR-223 could attenuate the inflammatory conditions in bovine endometritis by targeting NLRP3 in vivo and in vitro. ${ }^{73}$ In addition, it is worth noting that Helicobacter pylori (H. pylori) could modulate different immune responses in order to survive. In addition, Pachathundikandi et al reported that H. pylori could regulate NLRP3 expression in infected THP-1 monocytes. At the molecular level, the expression levels of hsa-miR-223-3p were significantly increased after $H$. pylori infection; this reduced NLRP3 expression and increased survival. ${ }^{74}$ Similarly, Wang et al demonstrated that miRNA-223 negatively regulated LPSinduced inflammatory responses by targeting NLRP3 in human dental pulp fibroblasts. ${ }^{75}$ Another study showed that the miR-223/NLRP3 axis was involved in stromal interaction molecule 1-mediated lung epithelial injury in influenza A virus-induced infection. ${ }^{76}$ Furthermore, the expression of miR-155 was up-regulated in Porphyromonas gingivalis-induced macrophages and the knockdown of miR-155 could reduce NLRP3 inflammasome-mediated pyroptosis. $^{77}$ These studies showed that miRNAs mainly participated in microbial infection by regulating NLRP3 inflammasome pathways. Other components of NLRP3 inflammasomes, such as caspase-1, GSDMD, and IL-1b, along with other inflammasomes such as AIM2, NLRP1, and NLRC4, should also be investigated further. Clinical studies are also needed to demonstrate their value in microbial infection.

As with miRNAs, only a few lncRNAs have been found to be involved in microbial infection by regulating the NFkB/NLRP3 pathway in vitro. For example, Deng et al showed that lncRNA Gm28309 was involved in regulating inflammation induced by Brucella. The overexpression of Gm28309, or the inhibition of miR-3068-5p, repressed p65 
phosphorylation and reduced the NLRP3 inflammasome, along with IL-1 $\beta$ and IL-18 secretion, in S2308 Brucella strain-induced RAW264.7 cells. Mechanistically, Gm28309 acts as a competing endogenous RNA (ceRNA) for miR$3068-5 \mathrm{p}$ to activate the NF- $\mathrm{kB}$ pathway by targeting $\kappa \mathrm{B}-\mathrm{Ras} 2$, an inhibitor of NF- $\mathrm{BB}$ signaling. Moreover, the number of intracellular Brucella is higher when Gm28309 is overexpressed or when miR-3068-5p or p65 is inhibited. ${ }^{78}$ In addition, IncRNA XIST is abnormally increased in bovine mastitis tissues and inflammatory mammary alveolar cell-T (MAC-T) cells, while silencing of lncRNA XIST significantly increases the expression of E. coli or $S$. aureusinduced pro-inflammatory cytokines. Mechanistically, IncRNA XIST is promoted by the activated NF- $\mathrm{BB}$ pathway and, in turn, generated a negative feedback loop to regulate the NF- $\mathrm{KB} / \mathrm{NLRP} 3$ inflammasome pathway, thus mediating inflammation in vitro. ${ }^{79}$ These two studies demonstrated that IncRNAs participated in Brucella, E. coli, or S. aureusinduced infection.

From the above research status, the ncRNAs inflammasome pathways in microbial infections are mainly concentrated on miRNAs and NLRP3 inflammasome, and the mechanisms are mainly concentrated on the signal 1 of NLPR3 inflammasome activation (Table 1). However, there is still a lack of research on inflammasomes regulated by circRNAs in microbial infection. Due to its circular structure and the fact that it is not easily degraded, circRNAs are more suitable as biomarkers; studies have shown that circRNA also participated in the inflammasome pathways, ${ }^{80}$ and deserved further research attention.

\section{The Roles of ncRNAs in Inflammasomes Activation in Infection-Induced Acute Organ Failure}

The Roles of ncRNAs in Inflammasomes Activation in ALI

An increasing number of studies have shown that the activation of inflammasomes played an important role in ALI, also known as acute respiratory distress syndrome (ARDS). The activation of inflammasomes in both the lung parenchyma and resident immune cells generates IL-1 $\beta$ and IL-18, both of which drive the cascade of lung inflammation forwards. ${ }^{81}$

Some studies have shown that ncRNAs directly targeted the components of inflammasomes in ALI (Table 1). The miR-223, the first miRNA found to target NLRP3, is the most studied miRNA in ALI. For example, Zhang et al reported that the secretion of EVs containing miR-223/142 was robustly enhanced and detectable in bronchoalveolar lavage fluid (BALF) and the serum of lung infection- associated ALI, and therefore has potential as a biomarker. The miR-223/142 mimics-enriched EVs have been shown to selectively target lung macrophages and suppress the inflammatory responses triggered by LPS or Klebsiella pneumoniae. Mechanistically, miR-223 and miR-142 synergistically suppressed the activation of the NLRP3 inflammasome by inhibiting NLRP3 and ASC in LPS-induced macrophages. ${ }^{19}$ At present, the most commonly used animal models of ALI are LPS-induced and trauma-induced as pathogen associated molecular patterns (PAMPs) and damage-associated molecular patterns (DAMPs)-induced ALI, respectively. The miR-223 was found to exert a treatment effect in trauma-induced ALI. Feng et al demonstrated that miR-223 expression was increased in the lungs of mitochondrial DAMPs (MTDs)induced mice or ARDS patients following trauma/ transfusion or following the physiological remission of ALI/ ARDS and miR-223 produced by Ly6G+ neutrophils. This could limit the number of Ly6G+ neutrophils and inhibit the activity of the NLRP3 inflammasome in a negative feedback manner to alleviate MTDs-induced ALI in miR-223 - $/+$ mice, thus regulating the pathogenesis of DAMPs-induced ALI. ${ }^{82}$ However, one study described low expression levels of miR-7b in NR8383 cells and lung tissues from LPS-induced mice, with no significant difference detected with regards miR-223. The authors also showed that silencing of the up-regulated IncMEG3 could alleviate LPS-induced ALI by acting as a molecular sponge for miR-7b to modulate NLRP3 directly in LPS-induced mice and LPS-induced mouse alveolar macrophage NR8383. ${ }^{83}$ This difference may be due to the induction of different stimuli; however, more research is needed to verify thus in clinical samples.

Furthermore, several studies have shown that miRNAs could target other components of inflammasomes or molecules upstream of inflammasomes in ALI except for NLRP3. TLR4, as an essential LPS signaling receptor, can activate NF- $\mathrm{KB}$ and participate in many inflammatory diseases. ${ }^{84}$ For instance, Yang et al suggested that NF- $\mathrm{KB}$, the NLRP3 inflammasome, and inflammatory factors, were reduced by miR-16 by directly targeting TLR 4 in LPSinduced ALI mice and A549 cells. ${ }^{85}$ In addition, Shikano et al conducted a microarray of EVs isolated from the BALF of LPS-induced ARDS mice and demonstrated increased expression levels of the miRNA-466 family. Mechanistically, miRNA-466g and 466m-5p affected the priming pathway by up-regulating pro-IL1b in macrophages, thus exacerbating inflammation in vitro. ${ }^{20}$ High 
mobility group box 1 (HMGB1), as a molecular of PAMPs, can be released after pyroptosis induced by the inflammasomes that are involved in various inflammatory diseases which in turn can activate inflammasomes. ${ }^{86,87}$ A recent study showed that miR-574-5p expression could be induced by the TLR4/NF- $\kappa$ B pathway upon LPS stimulation and in turn, miR-574-5p could suppress the inflammatory response, including the NF-kB signaling pathway and NLRP3 inflammasome activation by targeting HMGB1 in LPS-induced human pulmonary microvascular endothelial cells and ARDS mice, thus forming a negative feedback mechanism. ${ }^{88}$ These studies suggest that ncRNAs act on TLR4/NF-kB, pro-IL-1b, and HMGB1, and may also represent therapeutic targets.

Other studies have shown that miR-223 and miR-199a$3 p$ could attenuate LPS-induced cell injury in LPS-induced A549 cells. For example, miR-223 was shown to be involved in LPS-induced ALI by reducing inflammation. At the molecular level, miR-223 could suppress the NLRP3 inflammasome by targeting NLRP3 directly and the TLR4/NF- $\mathrm{BB}$ signaling pathway via RHOB in vitro. ${ }^{89}$ Activation of the NLRP1 has also been demonstrated in ALI; for example, microarray analysis showed that miR199a-3p was significantly downregulated in ALI lung tissues. The downregulation of miR-199a-3p has also been shown to induce the activation of NLRP1 and the cleavage of pro-IL-1 $\beta$ and pro-IL-18 by targeting NLRP1 directly, thus aggravating the inflammatory response in LPSinduced A549 and the human macrophage cell line U937. ${ }^{90}$ These studies demonstrated that ncRNAs could represent potential therapeutic targets for ALI.

The Roles of ncRNAs in Inflammasomes Activation in ALF ALF is a rapid and severe clinical syndrome characterized by the activation of intense inflammatory responses and the extensive death of hepatocytes, thus resulting in organ dysfunction and failure. Ongoing evidence describes a pivotal role for inflammasomes in this intense inflammatory response. ${ }^{91,92}$ Intraperitoneally injections of LPS and D-GalN are usually used to establish a mouse model of LPS/GalN-induced ALF, also known as endotoxin acute hepatitis $(\mathrm{EAH})$. So far, only a few studies have proved that ncRNAs have therapeutic effects on ALF, including miR-223-3p and miR-17 (Table 1). Calvente et al showed that the synthetic miR-223 analog miR-223 $3 p$ could attenuate LPS/D-GalN-induced liver injury by negatively regulating the NLRP3 inflammasome in LPS/D-GalNinduced EAH mice. ${ }^{93}$ In addition, TXNIP acts as a regulator of thioredoxin and can bind to NLRP3 via the leucine-rich repeat domain. A lack of TXNIP impairs activation of the NLRP3 inflammasome and subsequent IL-1 $\beta$ secretion. ${ }^{94}$ Liu et al demonstrated that the administration of adipose mesenchymal stem cell exosome (AMSC-Exo), co-localized with hepatic macrophages, significantly ameliorated ALF as determined by reduced serum levels of alanine aminotransferase and aspartate aminotransferase and hepatic inflammasome activation in LPS/GalN-induced ALF mice. Mechanically, AMSCderived exosomes containing miR-17 could alleviate LPS/GalN-induced ALF by the miR-17-mediated reduction of TXNIP/NLRP3 inflammasome activation in macrophages in vivo and in vitro. ${ }^{25}$ These two studies prove that miR-223-3p and AMSC-Exo with miR-17 may represent promising methods for the treatment of ALF.

The Roles of ncRNAs in Inflammasomes Activation in Sepsis-Induced AKI and Heart Dysfunction

Several studies have shown that ncRNAs participate in sepsis-induced AKI and sepsis-induced heart dysfunction by regulating inflammasomes; this process is also closely related to the amplification of excessive inflammation (Table 1). Autophagy dysfunction can lead to diseases with hyperinflammation and the excessive activation of NLRP3 inflammasome, thus acting as a major regulator of inflammasomes. More importantly, autophagy has a protective role in some inflammatory diseases associated with the NLRP3 inflammasome; ${ }^{95}$ this needs to be investigated further. A recent study showed that IncRNA ZFAS1 was highly expressed in sepsis-induced heart dysfunction in vivo and in an in vitro model. The knockdown of lncRNA ZFAS1 robustly abolished LPS-induced pyroptosis and attenuated the inhibition of autophagy in vivo and in vitro. Mechanistically, lncRNA ZFAS1 regulated autophagy and the NLRP3 inflammasome- mediated pyroptosis of cardiomyocytes by AMPK/mTOR signaling by sponging miR-590-3p. ${ }^{96}$

Several studies have focused on AKI from the perspective of ncRNAs and the activation of the NLRP3 inflammasome. For example, Tan et al demonstrated that IncRNA DLX6-AS1 was up-regulated in serum samples from septic AKI patients and in LPS-induced HK-2 cells. Mechanistically, IncRNA DLX6-AS1 mediated LPSmediated cytotoxicity and pyroptosis in $\mathrm{HK}-2$ via the miR-223-3p/NLRP3 axis. $^{97}$ In addition, a recent study showed that IncRNA RMRP was upregulated in sera from patients with AKI and in LPS-induced HK-2 cells 
and could promote inflammation by activating the NLRP3 inflammasome in vitro, thus providing a potential strategy for treating AKI. ${ }^{98}$ However, this study did not explain the specific mechanism by which lncRNA RMRP promoted NLRP3 activation. These two studies showed that lncRNA DLX6-AS1 and IncRNA RMRP had the potential to be biomarkers and might exert therapeutical effects. Furthermore, another study showed that IncRNA Kcnq1ot1 was upregulated in sC5b-9-induced podocytes, and that silencing Kcnq1ot1 could inhibit the effect of sC5b-9 on podocyte pyroptosis via a ceRNA mechanism by sponging miR-486a-3p which could bind directly to NLRP3. This might facilitate podocyte-targeted treatment for renal inflammatory diseases. ${ }^{99}$ Furthermore, ncRNAs/ inflammasomes pathways might represent the downstream targets for drugs in the treatment of AKI. For example, baicalin, a traditional Chinese medicine, could attenuate LPS-induced renal tubular epithelial cell injury by inhibiting the TXNIP/NLRP3 signaling pathway by increasing miR-223-3p expression in LPS-induced HK-2 cells. ${ }^{100}$

These studies indicate that ncRNAs are involved in multiple organ injury by regulating the activation of inflammasomes via diverse targets, including NLRP3, TLR4, pro-IL -1b, HMGB1, NLRP1 and TXNIP (Table 1). In addition, these studies showed that ncRNAs have therapeutic potential for microbial infection and tissue injury, although further research is needed to apply these in the clinic. Furthermore, with regards to the regulation of ncRNAs, the NLRP3 inflammasome pathway is the best investigated; very little is known about the involvement of other inflammasomes. Given the presumably large amounts of DNA released by dying cells during microbial infection and tissue injury, it is largely expected that the AIM2 inflammasome could play a role. Furthermore, the noncanonical inflammasome can also be activated in microbial infection and tissue injury. Further studies relating to ncRNAs in microbial infection and tissue injury should also feature inflammasomes.

\section{The Roles of ncRNAs in Inflammasomes Activation in Sterile Inflammatory Diseases}

Autoimmune diseases, autoinflammatory diseases (AIDs), and allergic diseases are common aseptic inflammatory diseases in recent years. These diseases are all caused by abnormal immune responses without microbial infection, but differences exist among these three diseases. Autoimmune diseases are characterized by an immune response that inappropriately targets the body's own healthy organs. ${ }^{101}$ AIDs are disorders of the innate immune system characterized by recurrent systematic inflammation and serious complications. Allergic diseases refer to a group of diseases caused by abnormal immune response of the body to harmless foreign substances, resulting in harm to the body. The main distinction between autoimmune diseases and AIDs is that autoimmune diseases are typically defined by a malfunction of the adaptive immune system while in AIDs the innate immune system is affected, which lacks autoantibodies and no antigen-specific T cells are involved. ${ }^{102}$ The difference of autoimmune diseases and allergic diseases is that an autoimmune disease is an immune response of the immune system against itself, while an allergic disease is an immune response against substances outside the body. ${ }^{103}$ In addition, GVHD is a significant challenge for all patients receiving allografts which deserves our attention. Although the etiology are different, abnormal immune responses can cause tissue and organ damage and threaten the health of body. In addition, it has been found that the activation of inflammasomes plays an important role in these diseases in recent years. ${ }^{103-105}$ Intriguingly, ncRNAs are emerging as the new inflammasome regulatory molecules. The following summarizes the role of ncRNAs in these sterile inflammatory diseases by regulating inflammasomes.

\section{The Roles of ncRNAs in Inflammasomes Activation in Autoimmune Diseases}

Autoimmune diseases were initially considered to be rare; however, rigorous epidemiological studies have now demonstrated that autoimmune diseases affected $3-5 \%$ of the population. ${ }^{101}$ More importantly, there are almost 100 distinct autoimmune diseases, some of which are organspecific such as primary biliary cirrhosis and some of which reflect a variety of immunological dysfunctions involving multiple organs, such as systemic lupus erythematosus (SLE); these conditions are associated with a poor prognosis and most of the current treatment drugs have certain side effects. ${ }^{106}$ Therefore, there is an urgent need to explore the molecular mechanisms of autoimmune diseases, the chronic and systemic inflammatory disorders characterized by dysfunction of the immune system, and ultimately the loss of immunological self-tolerance, to identify new therapeutic targets. Recent research found that active inflammasomes can trigger a series of inflammatory cascades and lead to the differentiation and 
polarization of naïve $\mathrm{T}$ cells and the secretion of various cytokines, thus inducing various types of autoimmune diseases, ${ }^{105}$ such as SLE, rheumatoid arthritis (RA), systemic sclerosis (SSc) and inflammatory bowel disease (IBD). ${ }^{107}$ Interestingly, an increasing number of studies have shown that ncRNAs regulated the activation of inflammasomes in autoimmune diseases (Table 1).

Several miRNAs are known to exert their function by directly targeting NLRP3 in autoimmune diseases. For example, miR-223 has been reported to be involved in various diseases by directly targeting NLRP3, including IBD and autoimmune myocarditis (EAM). Neudecker et al demonstrated that levels of miR-223 were increased in intestinal biopsies from patients with active IBD and in preclinical models of intestinal inflammation. The miR$223^{-/ y}$ mice presented with exacerbated myeloid-driven experimental colitis with heightened clinical, histopathological, and cytokine readouts. ${ }^{108}$ In addition, Chen et al reported that the transfer of miR-223-3p-overexpressing dendritic cells DCs protected mice against the development of EAM. Mechanistically, miR-223-3p inhibited the expression of the NLRP3 inflammasome expression, thus promoting the polarization of DCs towards a tolerogenic DC phenotype and regulatory $\mathrm{T}$ cell (Treg) generation. ${ }^{109}$ In addition, Tan et al reported that let-7f-5p was upregulated in bone marrow-derived mesenchymal stem cells (BMSCs) from systemic lupus erythematosus patients (SLE-BMSCs) and that let-7f-5p could attenuate inflammation in SLE-BMSCs by targeting NLRP3, at least in part, thus representing a promising therapy for SLE. ${ }^{110}$

In addition, $\mathrm{NF}-\kappa \mathrm{B}$ is a critical transcription factor that induces pro-IL-1 $\beta$ and upregulates the synthesis of NLRP3. ${ }^{70}$ TRAF6 and IRAK1 are key proteins in the TLR/NF-kB pathway. Zhang et al observed significant increases in paw swelling and index and ankle joint swelling in miR-146a knockout mice when compared with WT controls in a monosodium urate (MSU)-induced gouty arthritis model. At the molecular level, miR-146 knockout could increase inflammatory response and up-regulate NLRP3 expression partly by targeting TRAF6 and IRAK $1 .{ }^{22}$ In addition, Luo et al suggested that miR-155 expression levels were significantly increased in an in vivo model of psoriasis when compared with normal tissues; the silencing of miR-155 was confirmed to significantly reduce psoriasis-associated inflammatory responses and NLRP3/caspase-1 signaling in vitro. ${ }^{111}$

Furthermore, in vitro experiments have also demonstrated the therapeutic potential of miR-33 and miR-20a in autoimmune diseases. ROS can stimulate tissue inflammation and induce NLRP3 inflammasome activation as signal 2 (Figure 3). For instance, Xie et al reported that miR-33 impaired mitochondrial oxygen consumption rates, resulting in the accumulation of cellular ROS; this stimulated NLRP3 expression, caspase-1 activity, and IL$1 \beta$ secretion, in primary macrophages. The miR-33 levels and NLRP3 inflammasome activity were increased in peripheral blood monocytes from patients with rheumatoid arthritis (RA) compared with healthy donors, thus indicating that the miR-33/NLRP3 inflammasome pathway might be involved in RA development. ${ }^{24}$ In addition, RA is a heterogenic and systemic autoimmune disease characterized by synovitis and joint structural damage. As described earlier, TXNIP is a key protein that activates the NLRP3 inflammasome. $\mathrm{Xu}$ et al reported that the overexpression of miR-20a could reduce the activation of the NLRP3 inflammasome by targeting TXNIP directly in primary fibroblast-like synoviocytes (FLSs) isolated from adjuvant-induced arthritis (AA), which might be a treatment for RA. ${ }^{26}$ These studies showed that miRNAs had the potential to treat autoimmune diseases.

Crosstalk has been identified between inflammasomes and autophagy, both of which have been shown to be regulated by lncRNAs. Xue et al showed that lincRNACox 2 was also involved in EAM and identified a link between lincRNA-Cox2 and the crosstalk between inflammasomes and autophagy in macrophages and microglia. These authors found that lincRNA-Cox 2 bound directly to $\mathrm{NF}-\kappa \mathrm{B}$ p65 to promote $\mathrm{p} 65$ transfer from the cytoplasm to the nucleus, thus modulating the expression of the inflammasome sensor NLRP3 and the adaptor ASC. The knockdown of lincRNA-Cox2 inhibited the activation of inflammasomes and prevented the lincRNA-Cox2triggered activation of caspase-1, thus leading to reduced levels of IL-1 $\beta$ secretion and weakened TIR-domaincontaining adapter-inducing interferon- $\beta$ (TRIF) cleavage, thereby enhancing TRIF-mediated autophagy in LPSinduced microglial cells and BMDMs. These results identified a role for lncRNAs in NLRP3 inflammasome activation and autophagy, thus providing new opportunities for therapeutic intervention in neuroinflammation-dependent diseases. Furthermore, these authors also reported that the knockdown of lincRNA-Cox 2 provided a protective effect from experimental autoimmune encephalomyelitis, a notable increase in resting microglia, the inhibition of IL-1 $\beta$ secretion, and enhanced autophagy in the central nervous system. ${ }^{48}$ 
Collectively, these studies showed that ncRNAs regulated inflammasome pathways to play an important role in autoimmune diseases and provided a foundation for us to investigate the molecular mechanisms involved so that we can elucidate new forms of treatment. However, most of the current researches on the ncRNAs-inflammasome pathways in autoimmune diseases mainly focus on the NLRP3 inflammasome (Table 1). In recent years, it has been found that other inflammasomes also play an important role in autoimmune diseases. For instance, two ALR family members, the interferon (IFN)-inducible protein 16 (IFI16) and AIM2, have been linked to the pathogenesis of various autoimmune diseases where it triggers production of specific autoantibodies. Following interaction with cytoplasmic or nuclear nucleic acids, ALRs can form a functional inflammasome and produce IL-1b and IL-18 positively correlates with diverse autoimmune diseases severity. ${ }^{113}$ Therefore, targeting ALR sensors and their downstream pathways by ncRNAs also represents a promising alternative therapeutic approach for autoimmune diseases. In addition, literature relating to lncRNAs and relevant circRNAs are sparse. In view of the important regulatory role of IncRNAs and circRNAs in cell biological processes, there is an urgent need for researchers to focus on this field in future.

\section{The Roles of ncRNAs in Inflammasomes Activation in Autoinflammatory Diseases}

The name "autoinflammatory diseases (AIDs)" was proposed in 1999 by McDermott et al. ${ }^{114}$ Since its initial definition more than 30 new genes associated with autoinflammatory diseases have been identified, affecting different parts of the innate immune system. Depending on their molecular mechanism, AIDs can be separated into inflammasomopathies or IL-1 $\beta$-activation syndromes (Familial Mediterranean Fever (FMF), NLRP3-AID, Mevalonate kinase deficiency (MKD), deficiency of the IL-1 receptor antagonist, deficiency of IL-36 receptor antagonist), protein-folding disorders (TNF receptor-associated periodic syndrome), NF- $\kappa$ B-activation disorders (Blau syndrome), interferonopathies (Aicardi-Goutières syndromes), and other cytokine-signaling disorders and complementopathies (ie: paroxysmal nocturnal hemoglobinuria, atypical hemolytic uremic syndrome). In this review, we will mainly discuss inflammasomopathies. ${ }^{115}$ Among these inflammasomopathies, the most common diseases are FMF, NLRP3-AIDs (formerly known as Cryopyrin associated periodic syndromes - CAPS) and MKD which are all associated with inflammasomes hyperactivation. Additionally, these diseases are all belong to monogenic AIDs. Some polygenic or multifactorial AIDs have been also found, like systemic juvenile idiopathic arthritis (sJIA), which are more complex. They arise from permutations and combinations of common gene variants, where each variant alone confers only a small risk but together or with other extraneous influences becomes pathogenic. ${ }^{115}$ More importantly, sJIA was found due to a CASP1 variant causing inflammasomes hyperactivation recently. ${ }^{116}$ Dysregulation of inflammasomes and overproduction of IL-1 play a major role in the pathogenesis of these autoinflammatory diseases, including FMF, NLRP3-AIDs and sJIA which will be discussed below. ${ }^{117}$ Notably, ncRNAs as important regulators of inflammasomes have the potential as diagnostic biomarkers and therapeutic targets for autoinflammatory diseases. Advances in our understanding of the role of ncRNAs in the autoinflammatory diseases might result in new treatment strategies.

FMF is an inherited autosomal recessive autoinflammatory disease caused by mutations in the MEditerranean FeVer (MEFV) gene. MEFV gene encodes the protein pyrin, the core of pyrin inflammasomes, whose activation result in FMF. Notably, many FMF patients with similar genotypes can express different disease phenotypes. These differences in disease phenotype can be explained by modifying genes, epigenetic factors, or environmental effects. As important epigenetic modifiers, ncRNAs were found to be involved in the occurrence of FMF. For example, Wada et al observed specific miRNA patterns in different subgroups dependent on MEFV mutations. ${ }^{118}$ In addition, Akkaya-Ulum et al performed a miRNA array on whole blood RNA samples from 6 healthy controls (-/-), 6 FMF patients (M694V/M694V), 6 carriers who displayed the disease phenotype (M694V/-) and 6 healthy carriers (M694V/-), founding that miR-20a-5p was significantly up regulated whereas miR-197-3p was down regulated in homozygotes patients and both let-7d-3p and miR-574-3p were up regulated in heterozygote patients group. What's more, these miRNAs were found to be associated with inflammatory pathway related genes according to DAVID analysis. ${ }^{119}$ Similarly, Amarilyo et al performed the study to identify miRNAs expression profile in the peripheral blood of 10 M694V homozygous FMF patients and 10 healthy controls, founding four miRNAs were upregulated (miR-144-3p, miR-21-5p, miR-4454, and miR-451a), and three were downregulated (miR-107, let-7d-5p, and miR- 
148b-3p) compared to healthy controls. ${ }^{120}$ What's more, Hortu et al evaluated the role of fifteen miRNAs that were found to be associated with autoinflammatory diseases and have a part in immune response were evaluated in 51 patients with genetically diagnosed FMF who had clinical symptoms and 49 healthy volunteers. They found 11 miRNAs (miR-125a, miR-132, miR-146a, miR-155, miR-15a, miR-16, miR-181a, miR-21, miR-223, miR$26 \mathrm{a}$, and miR-34a) in the patient group were significantly down-regulated compared with the control group and 5 miRNAs (miR-132, miR-15a, miR-181a, miR-23b, miR26a) in the patients who took colchicine seemed to be upregulated and 5 miRNAs (miR-146a, miR-15a, miR-16, miR-26a, miR-34a) in the patients who took colchicine were significantly down-regulated, indicating the important role of miRNAs in the pathogenesis of FMF. ${ }^{121}$ Thus, a number of studies have proved that miRNAs are differentially expressed in FMF patients and controls, indicating that these miRNAs play an important role in FMF, which may be related to the activation of pyrin inflammasomes, but these studies have no further mechanisms and functional verification. Intriguingly, a study conducted by Akkaya-Ulum et al showed that miR-197-3p was decreased in FMF patients compared with controls and miR-197-3p could target IL1R1 gene and suppress cell migration, IL-1b secretion, caspase-1 activation and apoptosis in monocytes and synovial fibroblasts, indicating a promising treatment target in FMF. ${ }^{122}$ Therefore, in the future, more research is needed to explore the role of ncRNAs in FMF, finding potential biomarkers and therapeutic targets.

For other monogenic inflammasomopathies, research on ncRNAs is still vacant, such as CAPS, MKD, NLRP1 and NLRC4 inflammasome related AIDs. ${ }^{123,124}$ It has been found that targeting components of inflammasomes directly or inhibiting their activation has promising preventive or therapeutic effects on these inflammasomopathies. ${ }^{125}$ Therefore, discovered ncRNAs which can directly target components of inflammasomes (Figure 3), all have the potential as new targets for inflammasomes mediated AIDs. In addition, increasing studies have found ncRNAs can regulate other inflammasomes except NLRP3. For instance, miR-590-3p and miR-9a-5p could inhibit NLRP1 mediated inflammasomes activation in diabetic retinopathy and ischemic stroke-induced brain injury respectively by targeting NLRP1 directly. ${ }^{126,127}$ Moreover, IncRNA Neat1 associates with the NLRP3, NLRC4, and AIM2 inflammasomes in mouse macrophages to enhance their assembly and subsequent pro-caspase-1 processing. ${ }^{10}$ Future studies can verify the role of these potential ncRNAs in various inflammasomes mediated AIDs.

About sJIA, a kind of polygenic AIDs, is characterized by the overexpression of inflammasome-associated genes, leading to a dysregulation of the innate immune response, with a severe complication of macrophage activation syndrome (MAS). ${ }^{128}$ Notably, sJIA and recurrent MAS were found due to a CASP1 variant causing inflammasomes hyperactivation. ${ }^{116}$ Notably, two studies showed the correlation between miRNAs and sJIA. A microarray profile was performed in the plasma of patients with sJIA and healthy controls, founding 48 differential expressional miRNAs. Further they demonstrated that miR-26a was expressed specifically and highly in sJIA plasma and suggested that miR-26a might regulate the levels of cytokines in sJIA. ${ }^{129}$ In addition, it was found that miRNAs could contribute to monocyte/macrophage polarization with resulting consequences on MAS development. ${ }^{130}$ The study performed by Do et al showed that miR-125a-5p and miR-181c were elevated in active SJIA, significantly reducing macrophage CD163 expression through 2 distinct mechanisms. MiR-181 species directly targeted CD163 mRNA for degradation and overexpression of miR-181c inhibited CD163 anti-inflammatory responses to hemoglobin or HMGB1 complexes. In contrast, miR-125a-5p could function indirectly, as transcriptome analysis of miR-125a-5p overexpression identified "cytokinecytokine receptor interactions" as the most significantly repressed gene pathway, including decreased IL10RA, required for IL-10-mediated CD163 expression. Their data showed that miRNAs utilized multiple mechanisms to integrate well-characterized polarization phenotypes and regulate macrophage functional properties seen in SJIA. ${ }^{131}$ These studies show that ncRNAs have the potential as biomarkers and therapeutic targets. In the future, we need to pay attention to whether ncRNAs can regulate inflammasomes activation in sJIA.

What's more, other disease entities, such as Kawasaki disease and idiopathic recurrent pericarditis disease, gout or calcium pyrophosphate deposition disease are also believed to be related to polygenic AIDs. ${ }^{128}$ AIDs are an expanding spectrum of diseases, and more effort is needed to understand the pathogenesis.

The incidence of AIDs is rising, and diagnosis of AIDs is often based on clinical presentation and genetic testing and the timeline from onset to diagnosis takes up to 7.3 
years, highlighting the indisputable need to identify new treatment and diagnostic targets. ${ }^{115} \mathrm{NcRNAs}$, as important regulators in the activation of inflammasomes, are worthy of attention by researchers.

\section{The Roles of ncRNAs in Inflammasomes Activation in Allergic Diseases}

The incidence of allergic diseases is getting higher and higher, and it seriously affects people's quality of life. In recent years, it has been found that a variety of allergic diseases are related to the activation of inflammasomes, such as asthma, rhinitis, dermatitis and conjunctivitis, ${ }^{102,132}$ mainly about NLRP3 inflammasome. Moreover, NLRC4 was also found to be up-regulated in neutrophilic asthma, ${ }^{133}$ and Wang et al reported that AIM2 inflammasome was activation in murine allergic rhinitis (AR) and might mediate HMGB1 release. ${ }^{134}$ What's more, honey bee venom could induce AIM2 inflammasome activation in human keratinocytes, which might promote subsequent allergic responses. ${ }^{135}$ Therefore, targeting the activation of these inflammasomes helps to reduce the inflammatory response, thereby reducing the damage caused by allergies. Several studies showed that ncRNAs could regulate NLRP3 inflammasomes activation in AR as promising treatment targets. For example, Yu et al reported that they found a novel has_circ_0000520 was upregulated and miR-556-5p was down-regulated in AR patients and mice compared with controls, further proving that hsa_circ_0000520 ablation and miR-556-5p overexpression could suppress NLRP3-mediated cell pyroptosis to attenuate AR in mice models. Mechanistically, miR556-5p targeted both hsa_circ_0000520 and 3'untranslated region (3'-UTR) of NLRP3, and knockdown of hsa_circ_0000520 inactivated NLRP3-mediated epithelium pyroptosis through miR-556-5p in a ceRNAdependent manner. ${ }^{136}$ In addition, two studies showed miRNAs could attenuate the symptoms of AR in mice by regulating NLRP3 inflammasome. Wu et al showed that miR-224-5p was significantly decreased in nasal mucosa of AR mice and overexpression of miR-224-5p markedly attenuated sneezing and nasal rubbing events, IgE level in serum, and pathological alterations in nasal mucosa. Mechanistically, miR-224-5p treatment could remarkably suppress NLRP3 inflammasome activation and TLR4/ MyD88/NF-KB pathway in AR mice by targeting TLR4 directly. ${ }^{137}$ Similarly, the study conducted by Xiao et al showed that miR-133b ameliorated allergic inflammation and symptoms by targeting NLRP3 directly in AR mice. ${ }^{138}$ These three studies demonstrate the therapeutic potential of ncRNAs in AR by regulating NLRP3 inflammasomes activation.

There are only a few studies regarding ncRNAsinflammasome pathways in allergic diseases. As the activation of different inflammasomes has been continuously discovered to play an important role in allergic diseases, the role of ncRNAs in allergic diseases by regulating the activation of inflammasomes should also receive our attention.

\section{The Roles of ncRNAs in Inflammasomes Activation in GVHD}

GVHD is a significant challenge for all patients receiving allografts; improvement in the outcomes of GVHD has been the primary reason for the reduction in non-relapse mortality over time. ${ }^{139}$ Thus, it is critical that we gain a better understanding of the mechanisms that underlie immune rejection. A growing body of evidence suggests that immune tolerance is an ideal state for patients with GVHD. DCs, the most potent form of antigen-presenting cells (APCs), are central mediators in immune tolerance. Gaining an improved understanding of the molecular mechanisms involved in the induction of tolerance-DCs (tol-DCs) will help us to identify important targets for treatment. ${ }^{140}$ Intriguingly, several studies have demonstrated the crucial role for inflammasomes in the pathogenesis of GVHD and have provided a potential new avenue for the targeted therapy of this severe complication. ${ }^{141-143}$

Recent studies have shown that lncRNA NEAT1 and miR-155 were involved in tol-DCs by targeting NLRP3 inflammasomes (Table 1). Zhang et al found that lncRNA NEAT1 served as a ceRNA by sponging miR-3076-3p and could induce immune tolerance by targeting NLRP3 in DCs. The knockdown of lncRNA NEAT1 has also been shown to induce immune tolerance in mouse models of EAM and heart transplantation, thus indicating that lncRNA NEAT1 may be a promising therapeutic target for immune-related diseases. ${ }^{140}$ Moreover, Chen et al reported that the expression levels of miR-155 were upregulated in LPS-stimulated DCs and that miR-155 deficiency in the recipient could alleviate acute GVHD. At the molecular level, miR-155-deficient dendritic cells cause less severe GVHD by reducing migration and defective activation of the NLRP3 inflammasome. ${ }^{143}$ However, published research in this area is very limited and requires further attention. 
The Roles of ncRNAs in Inflammasomes Activation in Other Sterile Inflammatory Diseases

Several studies have shown that miRNAs and lncRNAs are involved in osteoarthritis (OA) by regulating NLRP3 inflammasomes (Table 1). For example, Zhou et al used a model of anterior cruciate ligament transection (ACLT)induced osteoarthritis (OA) and performed differential miRNAomics analysis of the synovial membrane in knee osteoarthritis (KOA) induced by ACLT in rats. These authors found that miR-233 regulated NLRP3 inflammasomes by directly suppressing the NLRP3 3'-UTR and was implicated in synovial membrane injury; this may be an important mechanism underlying the pathogenesis of KOA. ${ }^{144}$ Similarly, another study reported that sinomenine (SIN) could represent a potential target for the treatment of OA via a similar mechanism. In another study, Dong et al demonstrated that the administration of SIN markedly improved the degradation of articular cartilage in ACLTinduced OA mice, downregulated the levels of inflammatory cytokines and the protein expression of the components within the NLRP3 inflammasome, and upregulated the expression of miR-223-3p in OA mice and IL-1 $\beta$ stimulated chondrocytes. At the molecular level, the overexpression of miR-223-3p blocked apoptosis and the inflammatory response in chondrocytes by directly targeting NLRP3 in vitro, thus highlighting SIN as a potential target for the treatment of OA. ${ }^{145}$ These two studies both focused specifically on the miR-223/NLRP3 pathway.

In contrast, two other studies investigated the effect of lncRNA SNHG7 in OA. However, these authors arrived at the opposite conclusion. Wang et al demonstrated that the downregulation of IncRNA SNHG14 could inhibit the FSTL-1-mediated activation of the NLRP3 and TLR4/ $\mathrm{NF}-\kappa \mathrm{B}$ signaling pathways by targeting miR-124-3p in a rat model of OA by destabilizing the medial meniscus and IL-1 $\beta$-induced chondrocytes, thus attenuating inflammatory reactions in OA. Follistatin-like Protein 1 (FSTL1) is a TGF- $\beta$ super-family binding protein that can enhance the transcription of NLRP3 and procaspase 1 genes in the NLRP3 inflammasome. ${ }^{146}$ However, one recent study showed that lncRNA SNHG7 was significantly downregulated while miR-214-5p was significantly upregulated in OA patients and IL- $1 \beta$-induced primary chondrocytes; lncRNA SNHG7 were shown to prevent IL-1 $\beta$ induced OA by inhibiting the NLRP3 inflammasome and apoptosis via the miR-214-5p/PPARGC1B axis in vitro. Mechanistically, lncRNA SNHG7 serves as a competitive endogenous RNA by sponging miR-214-5p which targets PPARGC1B. ${ }^{147}$ PPARGC1B is able to activate $\operatorname{PPAR} \gamma$ and has been identified as an antiinflammatory protein in many diseases and functions by inhibiting the activation of $\mathrm{p} 38$ and the NF- $\mathrm{KB}$ pathway, as well as the NLRP3 inflammasome. Thus, the effect of lncRNA SNHG7 in OA needs further research attention.

Furthermore, miR-148a have been associated with alcoholic hepatitis (AH). Heo et al performed miRNA profiling and PCR analysis and identified a substantial reduction in the levels of miR-148a in the liver of patients with $\mathrm{AH}$, further highlighting that alcohol decreases the levels of miR-148a expression in hepatocytes via FoxO1, thus facilitating TXNIP overexpression and the activation of the NLRP3 inflammasome; these processes induced hepatocyte pyroptosis in vivo and in vitro. ${ }^{27}$

Collectively, these studies demonstrated that ncRNAs played an important role in $\mathrm{OA}$, and $\mathrm{AH}$, and are therefore promising therapeutic targets. However, most of the existing research has focused on the direct targeting of NLRP3 by miRNAs. Very few studies have investigated the potential role of lncRNAs and circRNAs.

\section{The Roles of ncRNAs in Inflammasomes Activation in Fibrosis Related Diseases}

Fibrosis can exert a severe effect on the function of organs, such as the lungs, liver, and heart, and are involved in the occurrence and development of a variety of diseases; excessive inflammation is a key factor in fibrosis. Excessive inflammation promotes fibrosis and aggravates the disease progression. In recent years, studies have shown that inflammasomes promote the progression of fibrosis and that regulating the activation of inflammasomes can improve the degree of fibrosis. ${ }^{148,149}$ It is noteworthy that ncRNAs can regulate the inflammasomes involved in fibrosis-related diseases, particularly hepatic fibrosis and lung fibrosis (Table 1).

There are several studies relating to the regulatory effects of ncRNAs on inflammasomes in hepatic fibrosis. For example, Spry1 (Protein sprouty homolog 1 ) has been shown to inhibit the ERK/NF-kB pathway while Smad2/3 has been shown to mediate NOX4-derived ROS production. ${ }^{150}$ Ning et al reported that circulating mir-21 was upregulated in patients with liver fibrosis. At the molecular level, mir-21 can mediate AngII-activated NLRP3 inflammasomes and resultant collagen production via the Spry1/ERK/NF-kB and Smad7/Smad2/3/NOX4 pathways by targeting Spry1 and Smad7 directly. Ang- 
(1-7) has been shown to protect against BDL or AngII infusion-induced hepatic fibrosis and inhibit mir-21 expression in vivo and in vitro. ${ }^{151}$ Moreover, it has been reported that miRNAs could also directly target GSDMD, the executive molecule for inflammasome activation. The overexpression of miR-379-5p blocks the arsenite-induced increase in GSDMD levels and the release of IL- $1 \beta$ by targeting GSDMD directly in arsenite-induced hepatocytes, thus revealing a new molecular mechanism for arsenite-induced liver damage, inflammation, and fibrosis. ${ }^{21}$ In addition, one lncRNA was found to exert a function in hepatic fibrosis by regulating the NF-kB /NLRP3 pathway. Silencing lnc-Lfar1 by a lentivirusshRNA was found to alleviate $\mathrm{CCl}_{4}$ - and BDL-induced proinflammatory M1 macrophage activation and NLRP3 inflammasome-mediated pyroptosis while in vitro experiments demonstrated that the knockdown of lnc-Lfar1 significantly suppressed the LPS- and IFN- $\gamma$-induced proinflammatory activation of macrophages and inhibited LPS/ATP- and LPS/Nigericin-induced NLRP3 inflammasome-mediated pyroptosis. Mechanistically, lnc-Lfar1 regulated the LPS- and IFN- $\gamma$-induced proinflammatory activation of macrophages through the NF-кB pathway, ${ }^{152}$ thus providing a potential therapeutic target against inflammation-related disorders, including hepatic fibrosis. Therefore, these studies indicated that ncRNAs regulation by suitable means had great potential for the development of therapy against hepatic inflammation/ fibrosis. In addition, increasing studies have showed that ncRNAs could attenuate liver fibrosis by regulating inflammation in animal model of hepatic fibrosis related diseases. For example, miR-378 expression was increased in livers of dietary obese mice and patients with nonalcoholic steatohepatitis (NASH) and liver-specific expression of miR-378 triggered the development of NASH and fibrosis by activating TNF $\alpha$ signaling. ${ }^{153}$ Moreover, miR223 was highly elevated in hepatocytes in high-fat diet (HFD)-fed mice and in human NASH samples. Genetic deletion of miR-223 induced a full spectrum of nonalcoholic fatty liver disease in long-term HFD-fed mice including steatosis, inflammation, fibrosis, and hepatocellular carcinoma. ${ }^{154}$ Additionally, enforcing the expression of circFBXW4 inhibited hepatic stellate cells activation, proliferation and induced apoptosis, attenuated mouse liver fibrogenesis injury and showed anti-inflammation effect. ${ }^{155}$ Furthermore, miR-155 KO mice are protected from alcohol-induced steatosis and inflammation. ${ }^{156}$ These ncRNAs have been proved at the animal level to play an important role in liver fibrosis-related diseases. In the future, new technologies and methods should be explored to target ncRNAs for clinical trials.

Furthermore, three miRNAs have been found to be involved in lung fibrosis by regulating the NLRP3 inflammasome pathway. Sun et al reported that AngII exacerbated bleomycin (BLM)-induced lung fibrosis in rats and elevated the levels of mir-21 and the NLRP3 inflammasome. In contrast, ACE2/Ang (1-7) has been shown to attenuate BLM-induced lung fibrosis, and decreased mir21 levels and the NLRP3 inflammasome. At the molecular level, mir-21 has been shown to mediate the inhibitory effect of ACE2/Ang (1-7) on the AngII-induced activation of the NLRP3 inflammasome by targeting Spry1 in lung fibroblasts. ${ }^{157}$ In addition, LOX1, a receptor for oxidized low-density lipoprotein (oxLDL) in endothelial cells, has been shown to be upregulated in many inflammationrelated pathophysiological events. ${ }^{158}$ Sun et al reported that the use of mesenchymal stem cell-derived exosomes (MenSC-Exo) significantly improved BLM-induced lung fibrosis and alveolar epithelial cell damage in mice. Mechanistically, MenSCs-Exo was able to transport miRNA Let-7 into recipient alveolar epithelial cells by directly targeting LOX1; this could influence activation of the NLRP3 inflammasome by affecting ROS and mtDNA damage. ${ }^{159}$ In addition, FOXO3a is a transcription factor of the $\mathrm{O}$ subclass in the forkhead family that inhibits pyroptosis by regulating inflammatory responses, including the inhibition of inflammasome activation. ${ }^{52,160}$ Zhou et al revealed that miR-96-targeted FOXO3a expression was identified to be involved in the regulation of NLRP3 inflammasome, serving as an important regulatory mechanism for pathogenesis of carbon black nanoparticle, a core constituent of air pollutants like fine particulate matter (PM2.5), -induced pulmonary fibrosis in vitro. ${ }^{23}$

The inflammatory response induced by inflammasomes is an important factor that can lead to fibrosis. Collectively, these studies have shown that targeting ncRNAs may reduce fibrosis by reducing the activation of inflammasomes and therefore represent potential therapeutic targets.

\section{The Roles of ncRNAs in Inflammasomes Activation in Other Diseases}

There are many other diseases that involve the activation of inflammasomes in a process that is regulated by 
ncRNAs, including diabetes, atherosclerosis, and cancer. Here, we summarize some of the important research findings related to these disease states so as to provide further inspiration for research targeting inflammation-related diseases.

For example, one study showed that lncRNA Gm15441 could suppress its own antisense transcript TXNIP thus reducing TXNIP-stimulated NLRP3 inflammasome activation in response to PPARA agonism and fasting; this provided evidence for a mechanism by which PPAR $\alpha$ attenuates the activation of inflammasomes in the liver in response to metabolic stress. ${ }^{31}$ Therefore, this lncRNA deserves further research attention. In addition, we should focus on other genes that can regulate inflammasome activation, including SIRT1. SIRT1 is a NAD ${ }^{+}$-dependent protein deacetylase; the induction of this gene is associated with multiple physiological processes, including cellular stress response and the immune response. ${ }^{161}$ Mao et al suggested that the transfection of exosomes from human mesenchymal stem cells (hMSCs) containing lncRNA KLF3-AS1 in rats, and the incubation of exosomes containing lncRNA KLF3-AS1 with hypoxic cardiomyocytes, both verified that the overexpression of IncRNA KLF3AS1 in exosomes could reduce the myocardial infarction (MI) area, decrease cellular apoptosis and pyroptosis, and attenuate the progression of MI. Mechanistically, IncRNA KLF3-AS1 is secreted in exosomes from hMSCs and acts as a ceRNA to sponge miR-138-5p to regulate SIRT1; this inhibits cell pyroptosis and attenuates the progression of MI. ${ }^{162}$ Another study showed that SIRT1 could alleviate hepatic ischemia-reperfusion injury via the miR-182mediated XBP1/NLRP3 pathway in in vivo assays carried out in mice in which SIRT had been specifically knocked out in the liver, and in in vitro assays. Mechanistically, the expression of miR-182 was positively regulated by SIRT1 in a mouse model of hepatic IR injury; miR-182 inhibited the expression of XBP1 by binding to the 3'-UTR of $\mathrm{XBP} 1$, thus providing a new perspective on the hepatoprotective mechanisms of SIRT1 in hepatic IR injury. ${ }^{163}$ In addition, AIM2 is known to act as a DNA sensor and can be activated by mtDNAs following the mitochondrial damage that occurs in many inflammatory diseases. Liang et al demonstrated that lncRNA MRG3 could regulate the AIM2 inflammasome in cerebral $\mathrm{I} / \mathrm{R}$ injury. The knockdown of up-regulated lncRNA MEG3 was shown to inhibit OGD/R-induced pyroptosis and inflammation. At the molecular level, the knockdown of lncRNA MEG3 inhibited the expression of AIM2 by sponging miR-485 in vitro. ${ }^{164}$ Furthermore, some studies have focused on circRNAs and the regulation of inflammasome pathways. In high glucose-induced vascular injury, Cheng et al reported that hsa_circ_0068087 was upregulated in HGinduced HUVECs and that the downregulation of hsa circ_0068087 ameliorated TLR4/NF-אB/NLRP3 inflammasome-mediated inflammation and endothelial cell dysfunction under high glucose conditions by sponging miR-197 in vitro. ${ }^{165}$

Collectively, through these studies in other nondiseases, the effect of other key molecules on ncRNAs regulating inflammasomes and its role in inflammatory diseases also pay more attention.

\section{The Crosstalk Among ROS, ncRNAs and Inflammasomes}

ROS can stimulate tissue inflammation and induce inflammasomes activation, which is one of the main damage factors in a variety of inflammatory diseases. It was recently shown that NLRP3 might directly sense the presence of ROS produced by normal or malfunctioning mitochondria or indirectly by other activators of NLRP3. ${ }^{112}$ For example, mtROS can promote the dissociation of thioredoxin from TXNIP and subsequently increase the interaction between TXNIP and NLRP3, leading to NLRP3 inflammasome activation and IL-1 $\beta$ maturation. ${ }^{166}$ In addition, inflammasomes activationinduced pyroptosis can increase the level of ROS further. ${ }^{167}$ AND then ROS activate NF-kB which in turn upregulates NLRP3 forming a negative feedback regulation. Notably, it has been discovered that ncRNAs are not only important regulators of the inflammasome pathway, but also have a close regulatory role with ROS in recent years. ${ }^{168,169}$ Therefore, knowing the crosstalk among ncRNAs, ROS, inflammasomes helps us further understand the therapeutic potential of ncRNAs in the inflammatory diseases.

ROS can cause differential expression of ncRNAs in a variety of diseases. ${ }^{163}$ Whether these ncRNAs can regulate the activation of inflammasomes needs further research. In addition, several studies showed that ncRNAs could regulate inflammasomes activation by regulate ROS. For instance, miR-200a low-expression increased Keap1 to block Nrf2 antioxidant pathway, and then enhanced ROS-driven TXNIP to activate NLRP3 inflammasome. ${ }^{170}$ Moreover, Liu et al reported that knockdown of IncRNA XIST could promote NLRP3 
inflammasome activation mediated pyroptosis by triggering miR-335/SOD2/ROS signal pathway in A549 cells. ${ }^{171}$ Furthermore, miR-33 impaired mitochondrial oxygen consumption rates, resulting in the accumulation of cellular ROS, thus stimulating NLRP3 inflammasomes activation. $^{24}$ However, these two studies did not discuss whether ROS was activated by increasing the binding of TXNIP and NLRP3 or NF-kB-mediated transcriptional enhancement of NLRP3. In addition, in addition to NLRP3 inflammasomes, the activation of other inflammasomes such as NLRP1 and AIM2 has also been reported to be involved in ROS. Therefore, ncRNAs may also be involved. ${ }^{172,173}$

Therefore, as ROS is an important mediator of inflammatory diseases and an important activator of inflammasomes, exploring the interaction between ncRNAs and them is more helpful to understand the therapeutic potential of ncRNAs in inflammatory diseases.

\section{Measures to Target ncRNAs Inflammasomes Pathways in Inflammatory Diseases}

Knowing how to target ncRNAs helps us to deeply explore the mechanisms of ncRNAs regulating inflammasomes and their role in inflammatory diseases, and can guide the transition from basic research to clinical practice.

MiRNAs levels can be modulated by utilizing transient transfections of miRNAs mimics or miRNAs antagonists. ${ }^{14}$ The most common strategies for controlling lncRNAs expression are antisense oligonucleotides (ASOs) and duplex RNAs. ${ }^{174}$ Notably, lncRNAs can act in either the nucleus or cytoplasm, therefore determining the subcellular localization is also important for understanding mechanism and designing strategies for manipulating lncRNAs expression and function. Duplex RNAs are a reliable approach for targeting lncRNAs in the cytoplasm and inhibiting gene expression. Whereas it has been shown that ASOs are more reliable gene silencing agents than duplex RNAs for RNAs that are localized to cell nuclei. The silencing of circRNAs is similar to IncRNAs and also depends on the distribution of nucleoplasm. ASOs may, therefore, be the silencing method of choice when a target RNA is thought to function in the nucleus, while duplex RNAs may be a better choice when a target is thought to function in the cytoplasm. $^{174}$
Notably, recent advancements in CRISPR/Cas9 technologies for gene knockout, knock-in, and point mutations may facilitate understanding the biological roles of ncRNAs and aid in the development of ncRNA-based targeted therapy. Gene-editing therapy can directly control the level of certain ncRNAs in cells, local tissues or organs which may yield less side effects than exogenously synthesized compounds, such as interfering RNAs and chemical drugs. ${ }^{175}$ For example, Piwecka et al used CRISPR-Cas9 technology to remove the locus encoding the circCdrlas from the mouse genome, founding out the effect of circCdr1 to normal brain function. ${ }^{176}$ Therefore, we believe that this budding and maturing technology can contribute to the exploration of the function of ncRNAs, drug discovery and the treatment of various diseases that were previously considered incurable.

These are currently the most commonly used methods for targeting ncRNAs, and more research is needed in the future to explore methods for targeting ncRNAs to promote the development of targeted ncRNAs drugs.

\section{Conclusion}

As a key component of the innate immune system and a trigger of adaptive immunity, inflammasomes play a crucial role in inflammatory diseases. Research in the field of ncRNAs is growing at a fast pace as these molecules have been found to perform a critical role in the regulating inflammasome pathways in inflammatory diseases. We hope that this review will help to provide a better understanding of the inflammasome pathways that are regulated by ncRNAs in the pathogenesis of inflammatory diseases.

However, a number of important questions remain unanswered. First, given the large number of ncRNAs differentially expression in inflammatory diseases and with the potential to regulate inflammasomes activation, it is crucial to identify the most important ncRNAs in various inflammatory diseases. Second, the field of inflammasomes is at its infancy in inflammatory diseases. It is urgent to explore which inflammasome plays the most important effect in these inflammatory diseases and whether ncRNAs can regulate different inflammasomes activation at the same time like lncRNA NEAT1. Third, the mechanisms of the interaction between ncRNAs and inflammasomes is not yet in-depth. Considering that lncRNAs and circRNAs can exert functionality by directly binding to DNA, RNA, and proteins and participate in 
transcriptional and post-transcriptional regulation, future studies should focus on specific ncRNAs and explore how they influence inflammasomes activation in specific inflammatory diseases. Fourth, there is still a lack of research on ncRNAs as markers in inflammatory diseases. To fully explore the potential of ncRNAs in inflammatory diseases diagnosis and targeted therapy, future research on the use of ncRNAs as biomarkers and therapeutics should not only focus on their identification and functional characterization, but also on using a large number of statistically significant patient cohorts to characterize diagnostic effect. Additionally, development of technologies for efficient detection of ncRNAs and their tissue-specific delivery methods are critical to the success of the diagnostics and therapeutics.

\section{Author Contributions}

All authors participated in drafting or revising of the manuscript, and approved the final manuscript. All authors contributed to data analysis, drafting or revising the article, have agreed on the journal to which the article will be submitted, gave final approval of the version to be published, and agree to be accountable for all aspects of the work.

\section{Funding}

This work was supported by the National Natural Science Foundation of China (82002021 to Ni Yang and 81971810 to Chun-Feng Liu), the Doctoral Start-up Foundation of Liaoning Province (2021-BS-107 to Tie-Ning Zhang), Liaoning Province Science and Technology Major Special Project (No.2020JH1/10300001 to Chun-Feng Liu), Shenyang Science and Technology Plan Project (20-205-4-002 to Chun-Feng Liu), and 345 Talent Program of Shengjing Hospital of China Medical University (M0691 to Tie-Ning Zhang).

\section{Disclosure}

The authors report no conflicts of interest in this work.

\section{References}

1. Mattick JS, Makunin IV. Non-coding RNA. Hum Mol Genet. 2006;15 (No suppl_1):R17-R29. doi:10.1093/hmg/dd1046

2. Marques-Rocha JL, Samblas M, Milagro FI, Bressan J, Martínez JA, Marti A. Noncoding RNAs, cytokines, and inflammation-related diseases. FASEB J. 2015;29(9):3595-3611. doi:10.1096/fj.14-260323
3. Lin CC, Shen YR, Chang CC, et al. Terminal uridyltransferase 7 regulates TLR4-triggered inflammation by controlling Regnase-1 mRNA uridylation and degradation. Nat Commun. 2021;12 (1):3878. doi:10.1038/s41467-021-24177-7

4. Kelley N, Jeltema D, Duan Y, He Y. The NLRP3 inflammasome: an overview of mechanisms of activation and regulation. Int J Mol Sci. 2019;20(13):3328. doi:10.3390/ijms20133328

5. Menon MP, Hua KF. The long non-coding RNAs: paramount regulators of the NLRP3 inflammasome. Front Immunol. 2020;11:569524. doi:10.3389/fimmu.2020.569524

6. Eisenbarth SC, Flavell RA. Innate instruction of adaptive immunity revisited: the inflammasome. EMBO Mol Med. 2009;1 (2):92-98. doi:10.1002/emmm.200900014

7. Swanson KV, Deng M, Ting JP. The NLRP3 inflammasome: molecular activation and regulation to therapeutics. Nat Rev Immunol. 2019;19(8):477-489. doi:10.1038/s41577-019-0165-0

8. Bauernfeind F, Rieger A, Schildberg FA, Knolle PA, SchmidBurgk JL, Hornung V. NLRP3 inflammasome activity is negatively controlled by miR-223. $J$ Immunol 2012;189 (8):4175-4181. doi:10.4049/jimmunol.1201516

9. Haneklaus M, Gerlic M, Kurowska-Stolarska M, et al. Cutting edge: miR-223 and EBV miR-BART15 regulate the NLRP3 inflammasome and IL-1 $\beta$ production. $J$ Immunol. 2012;189 (8):3795-3799. doi:10.4049/jimmunol.1200312

10. Zhang P, Cao L, Zhou R, Yang X, Wu M. The lncRNA Neat1 promotes activation of inflammasomes in macrophages. Nat Commun. 2019;10(1):1495. doi:10.1038/s41467-019-09482-6

11. Lian C, Sun J, Guan W, et al. Circular RNA circHIPK3 activates macrophage NLRP3 inflammasome and TLR4 pathway in gouty arthritis via sponging miR-561 and miR-192. Inflammation. 2021. doi:10.1007/s10753-021-01483-2

12. Slack FJ, Chinnaiyan AM. The role of non-coding RNAs in oncology. Cell. 2019;179(5):1033-1055. doi:10.1016/j. cell.2019.10.017

13. Cai Y, Yu X, Hu S, Yu J. A brief review on the mechanisms of miRNA regulation. Genomics Proteomics Bioinformatics. 2009;7 (4):147-154. doi:10.1016/s1672-0229(08)60044-3

14. Lu TX, Rothenberg ME. MicroRNA. J Allergy Clin Immunol. 2018;141(4):1202-1207. doi:10.1016/j.jaci.2017.08.034

15. Barwari T, Joshi A, Mayr M. MicroRNAs in cardiovascular disease. $J$ Am Coll Cardiol. 2016;68(23):2577-2584. doi:10.1016/j.jacc.2016.09.945

16. Sun Z, Shi K, Yang S, et al. Effect of exosomal miRNA on cancer biology and clinical applications. Mol Cancer. 2018;17(1):147. doi:10.1186/s12943-018-0897-7

17. van den Berg MMJ, Krauskopf J, Ramaekers JG, Kleinjans JCS, Prickaerts J, Briedé JJ. Circulating microRNAs as potential biomarkers for psychiatric and neurodegenerative disorders. Prog Neurobiol. 2020;185:101732. doi:10.1016/j.pneurobio.20 19.101732

18. Rupaimoole R, Slack FJ. MicroRNA therapeutics: towards a new era for the management of cancer and other diseases. Nat Rev Drug Discov. 2017;16(3):203-222. doi:10.1038/nrd.2016.246

19. Zhang D, Lee $H$, Wang $X$, et al. A potential role of microvesicle-containing miR-223/142 in lung inflammation. Thorax. 2019;74(9):865-874. doi:10.1136/thoraxjn1-2018-212994

20. Shikano S, Gon Y, Maruoka S, et al. Increased extracellular vesicle miRNA-466 family in the bronchoalveolar lavage fluid as a precipitating factor of ARDS. BMC Pulm Med. 2019;19 (1):110. doi:10.1186/s12890-019-0876-9

21. Li J, Xue J, Wang D, et al. Regulation of gasdermin D by miR$379-5 p$ is involved in arsenite-induced activation of hepatic stellate cells and in fibrosis via secretion of IL-1 $\beta$ from human hepatic cells. Metallomics. 2019;11(2):483-495. doi:10.1039/ c8mt00321a 
22. Zhang QB, Qing YF, Yin CC, et al. Mice with miR-146a deficiency develop severe gouty arthritis via dysregulation of TRAF 6, IRAK 1 and NALP3 inflammasome. Arthritis Res Ther. 2018;20(1):45. doi:10.1186/s13075-018-1546-7

23. Zhou L, Li P, Zhang M, et al. Carbon black nanoparticles induce pulmonary fibrosis through NLRP3 inflammasome pathway modulated by miR-96 targeted FOXO3a. Chemosphere. 2020;241:125075. doi:10.1016/j.chemosphere.2019.125075

24. Xie Q, Wei M, Zhang B, et al. MicroRNA-33 regulates the NLRP3 inflammasome signaling pathway in macrophages. Mol Med Rep. 2018;17(2):3318-3327. doi:10.3892/mmr.2017.8224

25. Liu Y, Lou G, Li A, et al. AMSC-derived exosomes alleviate lipopolysaccharide/d-galactosamine-induced acute liver failure by miR-17-mediated reduction of TXNIP/NLRP3 inflammasome activation in macrophages. EBioMedicine. 2018;36:140-150. doi:10.1016/j.ebiom.2018.08.054

26. Li XF, Shen WW, Sun YY, et al. MicroRNA-20a negatively regulates expression of NLRP3-inflammasome by targeting TXNIP in adjuvant-induced arthritis fibroblast-like synoviocytes. Joint Bone Spine. 2016;83(6):695-700. doi:10.1016/j. jbspin.2015.10.007

27. Heo MJ, Kim TH, You JS, Blaya D, Sancho-Bru P, Kim SG. Alcohol dysregulates miR-148a in hepatocytes through FoxO1, facilitating pyroptosis via TXNIP overexpression. Gut. 2019;68 (4):708-720. doi:10.1136/gutjnl-2017-315123

28. Kopp F, Mendell JT. Functional classification and experimental dissection of long noncoding RNAs. Cell. 2018;172(3):393-407. doi:10.1016/j.cell.2018.01.011

29. Kapranov P, Cheng J, Dike S, et al. RNA maps reveal new RNA classes and a possible function for pervasive transcription. Science. 2007;316(5830):1484-1488. doi:10.1126/science.1138341

30. Yang Z, Jiang S, Shang J, et al. LncRNA: shedding light on mechanisms and opportunities in fibrosis and aging. Ageing Res Rev. 2019;52:17-31. doi:10.1016/j.arr.2019.04.001

31. Brocker $\mathrm{CN}$, Kim D, Melia $\mathrm{T}$, et al. Long non-coding RNA Gm15441 attenuates hepatic inflammasome activation in response to PPARA agonism and fasting. Nat Commun. 2020;11(1):5847. doi:10.1038/s41467-020-19554-7

32. Huang JZ, Chen M, Chen D, et al. A peptide encoded by a putative lncRNA HOXB-AS3 suppresses colon cancer growth. Mol Cell. 2017;68(1):171-84.e6. doi:10.1016/j.molcel.2017.09.015

33. Bhatta A, Atianand M, Jiang Z, Crabtree J, Blin J, Fitzgerald KA. A mitochondrial micropeptide is required for activation of the Nlrp3 inflammasome. J Immunol 2020;204(2):428-437. doi:10.4049/jimmunol.1900791

34. Sun M, Nie F, Wang Y, et al. LncRNA HOXA11-AS promotes proliferation and invasion of gastric cancer by scaffolding the chromatin modification factors PRC2, LSD1, and DNMT1. Cancer Res. 2016;76(21):6299-6310. doi:10.1158/0008-5472. Can-16-0356

35. Wu H, Qiao F, Zhao Y, et al. Downregulation of long non-coding RNA FALEC inhibits gastric cancer cell migration and invasion through impairing ECM1 expression by exerting its enhancer-like function. Front Genet. 2019;10:255. doi:10.3389/ fgene.2019.00255

36. Lin Y, Wang S, Gao L, et al. Oscillating lncRNA Platr4 regulates NLRP3 inflammasome to ameliorate nonalcoholic steatohepatitis in mice. Theranostics. 2021;11(1):426-444. doi:10.7150/ thno. 50281

37. Wang S, Lin Y, Li F, et al. An NF- $\kappa B$-driven IncRNA orchestrates colitis and circadian clock. Sci Adv. 2020;6(42):eabb5202. doi:10.1126/sciadv.abb5202

38. Cai LJ, Tu L, Huang XM, et al. LncRNA MALAT1 facilitates inflammasome activation via epigenetic suppression of $\mathrm{Nrf} 2$ in Parkinson's disease. Mol Brain. 2020;13(1):130. doi:10.1186/ s13041-020-00656-8
39. Pefanis E, Wang J, Rothschild G, et al. RNA exosome-regulated long non-coding RNA transcription controls super-enhancer activity. Cell. 2015;161(4):774-789. doi:10.1016/j.cell.2015.04.034

40. Huang Z, Du G, Huang X, et al. The enhancer RNA lnc-SLC4A1-1 epigenetically regulates unexplained recurrent pregnancy loss (URPL) by activating CXCL8 and NF-kB pathway. EBioMedicine. 2018;38:162-170. doi:10.1016/j.ebiom.2018.11.015

41. Ounzain S, Pedrazzini T. The promise of enhancer-associated long noncoding RNAs in cardiac regeneration. Trends Cardiovasc Med. 2015;25(7):592-602. doi:10.1016/j.tcm.2015.01.014

42. Yap K, Mukhina S, Zhang G, et al. Repeat-enriched RNA assembles a nuclear compartment to control alternative splicing and promote cell survival. Mol Cell. 2018;72(3):525-40.e13. doi:10.1016/j.molcel.2018.08.041

43. Yan B, Yao J, Liu JY, et al. IncRNA-MIAT regulates microvascular dysfunction by functioning as a competing endogenous RNA. Circ Res. 2015;116(7):1143-1156. doi:10.1161/circresaha.116.305510

44. Chi K, Geng X, Liu C, et al. LncRNA-HOTAIR promotes endothelial cell pyroptosis by regulating the miR-22/NLRP3 axis in hyperuricaemia. J Cell Mol Med. 2021;25:8504-8521. doi: $10.1111 / \mathrm{jcmm} .16812$

45. Zhou Y, Li T, Chen Z, Huang J, Qin Z, Li L. Overexpression of IncRNA TUG1 alleviates NLRP3 inflammasome-mediated cardiomyocyte pyroptosis through targeting the miR-186-5p/XIAP axis in coronary microembolization-induced myocardial damage. Front Immunol. 2021;12:637598. doi:10.3389/fimmu.2021.637598

46. Wang C, Yang Y, Zhang G, et al. Long noncoding RNA EMS connects c-Myc to cell cycle control and tumorigenesis. Proc Natl Acad Sci U S A. 2019;116(29):14620-14629. doi:10.1073/ pnas. 1903432116

47. Dimartino D, Colantoni A, Ballarino $\mathrm{M}$, et al. The long non-coding RNA lnc-31 interacts with Rock1 mRNA and mediates its YB-1-dependent translation. Cell Rep. 2018;23 (3):733-740. doi:10.1016/j.celrep.2018.03.101

48. Xue Z, Zhang Z, Liu H, et al. lincRNA-Cox2 regulates NLRP3 inflammasome and autophagy mediated neuroinflammation. Cell Death Differ. 2019;26(1):130-145. doi:10.1038/s41418-018-0105-8

49. Li H, Xu JD, Fang XH, et al. Circular RNA circRNA_000203 aggravates cardiac hypertrophy via suppressing miR-26b-5p and miR-140-3p binding to Gata4. Cardiovasc Res. 2020;116 (7):1323-1334. doi:10.1093/cvr/cvz215

50. Su Y, Lv X, Yin W, et al. CircRNA Cdrlas functions as a competitive endogenous RNA to promote hepatocellular carcinoma progression. Aging. 2019;11(19):8183-8203. doi:10.18632/ aging. 102312

51. Bian Y, Pang P, Li X, et al. CircHelz activates NLRP3 inflammasome to promote myocardial injury by sponging miR-133a-3p in mouse ischemic heart. J Mol Cell Cardiol. 2021;158:128-139. doi:10.1016/j.yjmcc.2021.05.010

52. Yan B, Zhang Y, Liang C, et al. Stem cell-derived exosomes prevent pyroptosis and repair ischemic muscle injury through a novel exosome/circHIPK3/ FOXO3a pathway. Theranostics. 2020;10(15):6728-6742. doi:10.7150/thno.42259

53. Zhang Z, Zhang T, Feng R, Huang H, Xia T, Sun C. circARF3 alleviates mitophagy-mediated inflammation by targeting miR-103/TRAF3 in mouse adipose tissue. Mol Ther Nucleic Acids. 2019;14:192-203. doi:10.1016/j.omtn.2018.11.014

54. Yang F, Hu A, Li D, et al. Circ-HuR suppresses HuR expression and gastric cancer progression by inhibiting CNBP transactivation. Mol Cancer. 2019;18(1):158. doi:10.1186/ s12943-019-1094-z

55. Barbagallo D, Caponnetto A, Cirnigliaro $\mathrm{M}$, et al. CircSMARCA5 inhibits migration of glioblastoma multiforme cells by regulating a molecular axis involving splicing factors SRSF1/SRSF3/PTB. Int J Mol Sci. 2018;19(2):480. doi:10.3390/ ijms 19020480 
56. Sun YM, Wang WT, Zeng ZC, et al. circMYBL2, a circRNA from MYBL2, regulates FLT3 translation by recruiting PTBP1 to promote FLT3-ITD AML progression. Blood. 2019;134 (18):1533-1546. doi:10.1182/blood.2019000802

57. Du WW, Yang W, Liu E, Yang Z, Dhaliwal P, Yang BB. Foxo3 circular RNA retards cell cycle progression via forming ternary complexes with p21 and CDK2. Nucleic Acids Res. 2016;44 (6):2846-2858. doi:10.1093/nar/gkw027

58. Legnini I, Di Timoteo G, Rossi F, et al. Circ-ZNF609 is a circular RNA that can be translated and functions in myogenesis. Mol Cell. 2017;66(1):22-37.e9. doi:10.1016/j.molcel.2017.02.017

59. Wang Y, Liu X, Shi H, et al. NLRP3 inflammasome, an immune-inflammatory target in pathogenesis and treatment of cardiovascular diseases. Clin Transl Med. 2020;10(1):91-106. doi:10.1002/ctm2.13

60. Singer M, Deutschman CS, Seymour CW, et al. The third international consensus definitions for sepsis and septic shock (Sepsis-3). JAMA. 2016;315(8):801-810. doi:10.1001/ jama.2016.0287

61. Rocheteau P, Chatre L, Briand D, et al. Sepsis induces long-term metabolic and mitochondrial muscle stem cell dysfunction amenable by mesenchymal stem cell therapy. Nat Commun. 2015;6:10145. doi:10.1038/ncomms 10145

62. Huang M, Cai S, Su J. The pathogenesis of sepsis and potential therapeutic targets. Int J Mol Sci. 2019;20(21):5376. doi:10.3390/ ijms20215376

63. Zhang Y, Li X, Grailer JJ, et al. Melatonin alleviates acute lung injury through inhibiting the NLRP3 inflammasome. $J$ Pineal Res. 2016;60(4):405-414. doi:10.1111/jpi.12322

64. Zhan C, Lin G, Huang Y, Wang Z, Zeng F, Wu S. A dopamine-precursor-based nanoprodrug for in-situ drug release and treatment of acute liver failure by inhibiting NLRP3 inflammasome and facilitating liver regeneration. Biomaterials. 2021;268:120573. doi:10.1016/j.biomaterials.2020.120573

65. Lin Q, Li S, Jiang N, et al. PINK1-parkin pathway of mitophagy protects against contrast-induced acute kidney injury via decreasing mitochondrial ROS and NLRP3 inflammasome activation. Redox Biol. 2019;26:101254. doi:10.1016/j.redox.2019.101254

66. Li N, Zhou H, Wu H, et al. STING-IRF3 contributes to lipopolysaccharide-induced cardiac dysfunction, inflammation, apoptosis and pyroptosis by activating NLRP3. Redox Biol. 2019;24:101215. doi:10.1016/j.redox.2019.101215

67. Lou J, Wang Y, Zhang Z, Qiu W. MiR-20b inhibits mycobacterium tuberculosis induced inflammation in the lung of mice through targeting NLRP3. Exp Cell Res. 2017;358(2):120-128. doi:10.1016/j.yexcr.2017.06.007

68. Long FQ, Kou CX, Li K, Wu J, Wang QQ. MiR-223-3p inhibits rTp17-induced inflammasome activation and pyroptosis by targeting NLRP3. J Cell Mol Med. 2020;24(24):14405-14414. doi: $10.1111 / \mathrm{jcmm} .16061$

69. He Y, Hara H, Núñez G. Mechanism and regulation of NLRP3 inflammasome activation. Trends Biochem Sci. 2016;41 (12):1012-1021. doi:10.1016/j.tibs.2016.09.002

70. Martinon F, Mayor A, Tschopp J. The inflammasomes: guardians of the body. Annu Rev Immunol. 2009;27:229-265. doi:10.1146/ annurev.immunol.021908.132715

71. Xue Z, Xi Q, Liu H, et al. miR-21 promotes NLRP3 inflammasome activation to mediate pyroptosis and endotoxic shock. Cell Death Dis. 2019;10(6):461. doi:10.1038/s41419-019-1713-z

72. Lee H, Groot M, Pinilla-Vera M, Fredenburgh LE, Jin Y. Identification of miRNA-rich vesicles in bronchoalveolar lavage fluid: insights into the function and heterogeneity of extracellular vesicles. J Control Release. 2019;294:43-52. doi:10.1016/j. jconrel.2018.12.008
73. Zhao G, Jiang K, Yang Y, et al. The potential therapeutic role of miR-223 in bovine endometritis by targeting the NLRP3 inflammasome. Front Immunol. 2018;9:1916. doi:10.3389/ fimmu.2018.01916

74. Pachathundikandi SK, Backert S. Helicobacter pylori controls NLRP3 expression by regulating hsa-miR-223-3p and IL-10 in cultured and primary human immune cells. Innate Immun. 2018;24(1):11-23. doi:10.1177/1753425917738043

75. Wang D, Sun S, Xue Y, et al. MicroRNA-223 negatively regulates LPS-induced inflammatory responses by targeting NLRP3 in human dental pulp fibroblasts. Int Endod J. 2021;54 (2):241-254. doi:10.1111/iej.13413

76. Liu CC, Miao Y, Chen RL, et al. STIM1 mediates IAV-induced inflammation of lung epithelial cells by regulating NLRP3 and inflammasome activation via targeting miR-223. Life Sci. 2021;266:118845. doi:10.1016/j.lfs.2020.118845

77. Li C, Yin W, Yu N, et al. miR-155 promotes macrophage pyroptosis induced by Porphyromonas gingivalis through regulating the NLRP3 inflammasome. Oral Dis. 2019;25(8):2030-2039. doi:10.1111/odi.13198

78. Deng X, Guo J, Sun Z, et al. Brucella-induced downregulation of lncRNA Gm28309 triggers macrophages inflammatory response through the miR-3068-5p/NF- $\mathrm{BB}$ pathway. Front Immunol. 2020;11:581517. doi:10.3389/fimmu.2020.581517

79. Ma M, Pei Y, Wang X, Feng J, Zhang Y, Gao MQ. LncRNA XIST mediates bovine mammary epithelial cell inflammatory response via NF-kB/NLRP3 inflammasome pathway. Cell Prolif. 2019;52(1):e12525. doi:10.1111/cpr.12525

80. Li M, Hua Q, Shao Y, et al. Circular RNA circBbs9 promotes PM (2.5)-induced lung inflammation in mice via NLRP3 inflammasome activation. Environ Int. 2020;143:105976. doi:10.1016/j. envint.2020.105976

81. McVey MJ, Steinberg BE, Goldenberg NM. Inflammasome activation in acute lung injury. Am J Physiol Lung Cell Mol Physiol. 2020. doi:10.1152/ajplung.00303.2020

82. Feng Z, Qi S, Zhang Y, et al. Ly6G+ neutrophil-derived miR-223 inhibits the NLRP3 inflammasome in mitochondrial DAMP-induced acute lung injury. Cell Death Dis. 2017;8(11): e3170. doi:10.1038/cddis.2017.549

83. Liao H, Zhang S, Qiao J. Silencing of long non-coding RNA MEG3 alleviates lipopolysaccharide-induced acute lung injury by acting as a molecular sponge of microRNA-7b to modulate NLRP3. Aging. 2020;12(20):20198-20211. doi:10.18632/ aging. 103752

84. Cao C, Yin C, Shou S, et al. Ulinastatin protects against LPSinduced acute lung injury by attenuating TLR4/NF- $\mathrm{kB}$ pathway activation and reducing inflammatory mediators. Shock. 2018;50 (5):595-605. doi:10.1097/shk.0000000000001104

85. Yang Y, Yang F, Yu X, et al. miR-16 inhibits NLRP3 inflammasome activation by directly targeting TLR4 in acute lung injury. Biomed Pharmacother. 2019;112:108664. doi:10.1016/j. biopha.2019.108664

86. Jiao B, Guo S, Yang X, et al. The role of HMGB1 on TDIinduced NLPR3 inflammasome activation via ROS/NF- $\mathrm{kB}$ pathway in HBE cells. Int Immunopharmacol. 2021;98:107859. doi:10.1016/j.intimp.2021.107859

87. Lu B, Nakamura $\mathrm{T}$, Inouye $\mathrm{K}$, et al. Novel role of PKR in inflammasome activation and HMGB1 release. Nature. 2012;488(7413):670-674. doi:10.1038/nature11290

88. He B, Zhou W, Rui Y, Liu L, Chen B, Su X. MicroRNA-574-5p attenuates acute respiratory distress syndrome by targeting HMGB1. Am J Respir Cell Mol Biol. 2021;64(2):196-207. doi:10.1165/rcmb.2020-0112OC 
89. Yan Y, Lu K, Ye T, Zhang Z. MicroRNA-223 attenuates LPS-induced inflammation in an acute lung injury model via the NLRP3 inflammasome and TLR4/NF- $\mathrm{BB}$ signaling pathway via RHOB. Int J Mol Med. 2019;43(3):1467-1477. doi:10.3892/ ijmm.2019.4075

90. Chen Z, Dong WH, Chen Q, Li QG, Qiu ZM. Downregulation of miR-199a-3p mediated by the CtBP2-HDAC1-FOXP3 transcriptional complex contributes to acute lung injury by targeting NLRP1. Int J Biol Sci. 2019;15(12):2627-2640. doi:10.7150/ ijbs.37133

91. Rolando N, Wade J, Davalos M, Wendon J, Philpott-Howard J, Williams R. The systemic inflammatory response syndrome in acute liver failure. Hepatology. 2000;32(4 Pt 1):734-739. doi:10.1053/jhep.2000.17687

92. Antoniades CG, Berry PA, Wendon JA, Vergani D. The importance of immune dysfunction in determining outcome in acute liver failure. $J$ Hepatol. 2008;49(5):845-861. doi:10.1016/j. jhep.2008.08.009

93. Jimenez Calvente C, Del Pilar H, Tameda M, Johnson CD, Feldstein AE. MicroRNA $2233 p$ negatively regulates the NLRP3 inflammasome in acute and chronic liver injury. Mol Ther. 2020;28(2):653-663. doi:10.1016/j.ymthe.2019.09.013

94. Zhou R, Tardivel A, Thorens B, Choi I, Tschopp J. Thioredoxininteracting protein links oxidative stress to inflammasome activation. Nat Immunol. 2010;11(2):136-140. doi:10.1038/ ni. 1831

95. Biasizzo M, Kopitar-Jerala N. Interplay between NLRP3 inflammasome and autophagy. Front Immunol. 2020;11:591803. doi:10.3389/fimmu.2020.591803

96. Liu JJ, Li Y, Yang MS, Chen R, Cen CQ. SP1-induced ZFAS1 aggravates sepsis-induced cardiac dysfunction via miR-590-3p/ NLRP3-mediated autophagy and pyroptosis. Arch Biochem Biophys. 2020;695:108611. doi:10.1016/j.abb.2020.108611

97. Tan J, Fan J, He J, Zhao L, Tang H. Knockdown of LncRNA DLX6-AS1 inhibits HK-2 cell pyroptosis via regulating miR-2233p/NLRP3 pathway in lipopolysaccharide-induced acute kidney injury. J Bioenerg Biomembr. 2020;52(5):367-376. doi:10.1007/ s10863-020-09845-5

98. Zhang X, Huang Z, Wang Y, Wang T, Li J, Xi P. Long non-coding RNA RMRP contributes to sepsis-induced acute kidney injury. Yonsei Med J. 2021;62(3):262-273. doi:10.3349/ymj.2021.62.3.262

99. Zhang C, Gong Y, Li N, et al. Long noncoding RNA Kcnq1ot1 promotes sC5b-9-induced podocyte pyroptosis by inhibiting miR-486a-3p and upregulating NLRP3. Am J Physiol Cell Physiol. 2021;320(3):C355-C364. doi:10.1152/ajpcell.004 03.2020

100. Sun Y, Liu MW, Zhao YH, Lu YX, Wang YA, Tong CW. Baicalin attenuates lipopolysaccharide-induced renal tubular epithelial cell injury by inhibiting the TXNIP/NLRP3 signalling pathway via increasing miR-223-3p expression. J Biol Regul Homeost Agents. 2020;34(1):69-82. doi:10.23812/19-502-a

101. Wang L, Wang FS, Gershwin ME. Human autoimmune diseases: a comprehensive update. J Intern Med. 2015;278(4):369-395. doi:10.1111/joim. 12395

102. Masters SL, Simon A, Aksentijevich I, Kastner DL. Horror autoinflammaticus: the molecular pathophysiology of autoinflammatory disease. Annu Rev Immunol. 2009;27:621-668. doi:10.1146/ annurev.immunol.25.022106.141627

103. Xiao Y, Xu W, Su W. NLRP3 inflammasome: a likely target for the treatment of allergic diseases. Clin Experiment Allergy. 2018;48(9):1080-1091. doi:10.1111/cea.13190

104. Coll RC, Robertson AA, Chae JJ, et al. A small-molecule inhibitor of the NLRP3 inflammasome for the treatment of inflammatory diseases. Nat Med. 2015;21(3):248-255. doi:10.1038/ nm.3806
105. Shin JI, Lee KH, Joo YH, et al. Inflammasomes and autoimmune and rheumatic diseases: a comprehensive review. J Autoimmun. 2019;103:102299. doi:10.1016/j.jaut.2019.06.010

106. Iranzo A. Sleep and neurological autoimmune diseases. Neuropsychopharmacology. 2020;45(1):129-140. doi:10.1038/ s41386-019-0463-z

107. Shen HH, Yang YX, Meng X, et al. NLRP3: a promising therapeutic target for autoimmune diseases. Autoimmun Rev. 2018;17 (7):694-702. doi:10.1016/j.autrev.2018.01.020

108. Neudecker V, Haneklaus M, Jensen O, et al. Myeloid-derived miR-223 regulates intestinal inflammation via repression of the NLRP3 inflammasome. J Exp Med. 2017;214(6):1737-1752. doi:10.1084/jem.20160462

109. Chen L, Hou X, Zhang M, et al. MicroRNA-223-3p modulates dendritic cell function and ameliorates experimental autoimmune myocarditis by targeting the NLRP3 inflammasome. Mol Immunol. 2020;117:73-83. doi:10.1016/j.molimm.2019.10.027

110. Tan W, Gu Z, Leng J, et al. Let-7f-5p ameliorates inflammation by targeting NLRP3 in bone marrow-derived mesenchymal stem cells in patients with systemic lupus erythematosus. Biomed Pharmacother. 2019;118:109313. doi:10.1016/j.biopha.2019.109313

111. Luo Q, Zeng J, Li W, et al. Silencing of miR-155 suppresses inflammatory responses in psoriasis through inflammasome NLRP3 regulation. Int $J$ Mol Med. 2018;42(2):1086-1095. doi:10.3892/ijmm.2018.3677

112. Minutoli L, Puzzolo D, Rinaldi M, et al. ROS-mediated NLRP3 inflammasome activation in brain, heart, kidney, and testis ischemia/reperfusion injury. Oxid Med Cell Longev. 2016;2016:2183026. doi:10.1155/2016/2183026

113. Caneparo V, Landolfo S, Gariglio M, De Andrea M. The absent in melanoma 2-like receptor IFN-inducible protein 16 as an inflammasome regulator in systemic lupus erythematosus: the dark side of sensing microbes. Front Immunol. 2018;9:1180. doi:10.3389/ fimmu.2018.01180

114. McDermott MF, Aksentijevich I, Galon J, et al. Germline mutations in the extracellular domains of the $55 \mathrm{kDa}$ TNF receptor, TNFR1, define a family of dominantly inherited autoinflammatory syndromes. Cell. 1999;97(1):133-144. doi:10.1016/s00928674(00)80721-7

115. Krainer J, Siebenhandl S, Weinhäusel A. Systemic autoinflammatory diseases. J Autoimmun. 2020;109:102421. doi:10.1016/j. jaut.2020.102421

116. Jørgensen SE, Christiansen M, Høst C, et al. Systemic juvenile idiopathic arthritis and recurrent macrophage activation syndrome due to a CASP1 variant causing inflammasome hyperactivation. Rheumatology. 2020;59(10):3099-3105. doi:10.1093/rheumatology/keaa242

117. Tartey S, Kanneganti TD. Inflammasomes in the pathophysiology of autoinflammatory syndromes. J Leukoc Biol. 2020;107 (3):379-391. doi:10.1002/jlb.3mir0919-191r

118. Wada T, Toma T, Matsuda Y, et al. Microarray analysis of circulating microRNAs in familial Mediterranean fever. Mod Rheumatol. 2017;27(6):1040-1046. doi:10.1080/14397595.2017.1285845

119. Akkaya-Ulum YZ, Balci-Peynircioglu B, Karadag O, et al. Alteration of the microRNA expression profile in familial Mediterranean fever patients. Clin Exp Rheumatol. 2017;35(6):90-94.

120. Amarilyo G, Pillar N, Ben-Zvi I, et al. Analysis of microRNAs in familial Mediterranean fever. PLoS One. 2018;13(5):e0197829. doi:10.1371/journal.pone.0197829

121. Hortu HO, Karaca E, Sozeri B, et al. Evaluation of the effects of miRNAs in familial Mediterranean fever. Clin Rheumatol. 2019;38(3):635-643. doi:10.1007/s10067-017-3914-0

122. Akkaya-Ulum YZ, Akbaba TH, Tavukcuoglu Z, et al. Familial Mediterranean fever-related miR-197-3p targets IL1R1 gene and modulates inflammation in monocytes and synovial fibroblasts. Sci Rep. 2021;11(1):685. doi:10.1038/s41598-020-80097-4 
123. Herlin T, Jørgensen SE, Høst C, et al. Autoinflammatory disease with corneal and mucosal dyskeratosis caused by a novel NLRP1 variant. Rheumatology. 2020;59(9):2334-2339. doi:10.1093/rheumatology/kez612

124. Canna SW, de Jesus AA, Gouni S, et al. An activating NLRC4 inflammasome mutation causes autoinflammation with recurrent macrophage activation syndrome. Nat Genet. 2014;46 (10):1140-1146. doi:10.1038/ng.3089

125. Huang Y, Jiang $\mathrm{H}$, Chen $\mathrm{Y}$, et al. Tranilast directly targets NLRP3 to treat inflammasome-driven diseases. EMBO Mol Med. 2018;10 (4). doi:10.15252/emmm.201708689

126. Cao Y, Zhang H, Lu X, et al. Overexpression of microRNA-9a-5p ameliorates NLRP1 inflammasome-mediated ischemic injury in rats following ischemic stroke. Neuroscience. 2020;444:106-117. doi:10.1016/j.neuroscience.2020.01.008

127. Gu C, Draga D, Zhou C, et al. miR-590-3p inhibits pyroptosis in diabetic retinopathy by targeting NLRP1 and inactivating the NOX4 signaling pathway. Invest Ophthalmol Vis Sci. 2019;60 (13):4215-4223. doi:10.1167/iovs.19-27825

128. Malcova H, Milota T, Strizova Z, et al. Interleukin-1 blockade in polygenic autoinflammatory disorders: where are we now? Front Pharmacol. 2020;11:619273. doi:10.3389/fphar.2020.619273

129. Sun J, Feng M, Wu F, et al. Plasma miR-26a as a diagnostic biomarker regulates cytokine expression in systemic juvenile idiopathic arthritis. $J$ Rheumatol. 2016;43(8):1607-1614. doi:10.3899/jrheum.150593

130. Li D, Duan M, Feng Y, Geng L, Li X, Zhang W. MiR-146a modulates macrophage polarization in systemic juvenile idiopathic arthritis by targeting INHBA. Mol Immunol. 2016;77:205-212. doi:10.1016/j.molimm.2016.08.007

131. Do T, Tan R, Bennett M, et al. MicroRNA networks associated with active systemic juvenile idiopathic arthritis regulate CD163 expression and anti-inflammatory functions in macrophages through two distinct mechanisms. J Leukoc Biol. 2018;103 (1):71-85. doi:10.1002/jlb.2a0317-107r

132. Sebastião AI, Ferreira I, Brites G, Silva A, Neves BM, Teresa Cruz M. NLRP3 inflammasome and allergic contact dermatitis: a connection to demystify. Pharmaceutics. 2020;12(9):867. doi:10.3390/pharmaceutics 12090867

133. Tan HT, Hagner S, Ruchti F, et al. Tight junction, mucin, and inflammasome-related molecules are differentially expressed in eosinophilic, mixed, and neutrophilic experimental asthma in mice. Allergy. 2019;74(2):294-307. doi:10.1111/all.13619

134. Wang Y, Chen S, Yang PL, Chen JJ, Kong WJ, Wang YJ. AIM2 inflammasome activation may mediate high mobility group box 1 release in murine allergic rhinitis. Braz J Otorhinolaryngol. 2021. doi:10.1016/j.bjorl.2020.12.014

135. Dombrowski Y, Peric M, Koglin S, et al. Honey bee (Apis mellifera) venom induces AIM2 inflammasome activation in human keratinocytes. Allergy. 2012;67(11):1400-1407. doi:10.1111/all.12022

136. $\mathrm{Yu} \mathrm{X}$, Wang $\mathrm{M}$, Zhao $\mathrm{H}$, Cao $\mathrm{Z}$. Targeting a novel hsa_circ_0000520/miR-556-5p/NLRP3 pathway-mediated cell pyroptosis and inflammation attenuates ovalbumin (OVA)-induced allergic rhinitis (AR) in mice models. Inflam Res. 2021;70(6):719-729. doi:10.1007/s00011-021-01472-z

137. Wu J, Wu L, Zhang L, et al. Overexpression of miR-224-5p alleviates allergic rhinitis in mice via the TLR4/MyD88/NF- $\kappa \mathrm{B}$ pathway. Experiment Anim. 2021. doi:10.1538/expanim.20-0195

138. Xiao L, Jiang L, Hu Q, Li Y. MicroRNA-133b ameliorates allergic inflammation and symptom in murine model of allergic rhinitis by targeting Nlrp3. Cell Physiol Biochem. 2017;42 (3):901-912. doi:10.1159/000478645
139. Khoury HJ, Wang T, Hemmer MT, et al. Improved survival after acute graft-versus-host disease diagnosis in the modern era. Haematologica. 2017;102(5):958-966. doi:10.3324/ haematol.2016.156356

140. Zhang M, Zheng Y, Sun Y, et al. Knockdown of NEAT1 induces tolerogenic phenotype in dendritic cells by inhibiting activation of NLRP3 inflammasome. Theranostics. 2019;9(12):3425-3442. doi: $10.7150 /$ thno. 33178

141. Jankovic D, Ganesan J, Bscheider M, et al. The Nlrp3 inflammasome regulates acute graft-versus-host disease. $J$ Exp Med. 2013;210(10):1899-1910. doi:10.1084/jem.20130084

142. Zeiser R, Penack O, Holler E, Idzko M. Danger signals activating innate immunity in graft-versus-host disease. J Mol Med. 2011;89 (9):833-845. doi:10.1007/s00109-011-0767-x

143. Chen S, Smith BA, Iype J, et al. MicroRNA-155-deficient dendritic cells cause less severe GVHD through reduced migration and defective inflammasome activation. Blood. 2015;126 (1):103-112. doi:10.1182/blood-2014-12-617258

144. Zhou J, Zhao Y, Wu G, Lin B, Li Z, Liu X. Differential miRNAomics of the synovial membrane in knee osteoarthritis induced by bilateral anterior cruciate ligament transection in rats. Mol Med Rep. 2018;18(4):4051-4057. doi:10.3892/ mmr.2018.9385

145. Dong HC, Li PN, Chen CJ, et al. Sinomenine attenuates cartilage degeneration by regulating miR-223-3p/NLRP3 inflammasome signaling. Inflammation. 2019;42(4):1265-1275. doi:10.1007/ s10753-019-00986-3

146. Wang B, Li J, Tian F. Downregulation of 1ncRNA SNHG14 attenuates osteoarthritis by inhibiting FSTL-1 mediated NLRP3 and TLR4/NF- $\mathrm{B}$ pathway through miR-124-3p. Life Sci. 2021;270:119143. doi:10.1016/j.lfs.2021.119143

147. $\mathrm{Xu} \mathrm{J}$, Pei Y, Lu J, et al. LncRNA SNHG7 alleviates IL-1 $\beta$ induced osteoarthritis by inhibiting miR-214-5p-mediated PPARGC1B signaling pathways. Int Immunopharmacol. 2021;90:107150. doi:10.1016/j.intimp.2020.107150

148. Alegre F, Pelegrin P, Feldstein AE. Inflammasomes in liver fibrosis. Semin Liver Dis. 2017;37(2):119-127. doi:10.1055/ s-0037-1601350

149. Zhang H, Wang Z. Effect and regulation of the NLRP3 inflammasome during renal fibrosis. Front Cell Dev Biol. 2019;7:379. doi: $10.3389 /$ fcell.2019.00379

150. Ling $\mathrm{M}, \mathrm{Li} \mathrm{Y}, \mathrm{Xu} \mathrm{Y}$, et al. Regulation of miRNA-21 by reactive oxygen species-activated ERK/NF- $\kappa \mathrm{B}$ in arsenite-induced cell transformation. Free Radic Biol Med. 2012;52(9):1508-1518. doi:10.1016/j.freeradbiomed.2012.02.020

151. Ning ZW, Luo XY, Wang GZ, et al. MicroRNA-21 mediates angiotensin II-induced liver fibrosis by activating NLRP3 inflammasome/IL-1 $\beta$ axis via targeting Smad7 and Spry1. Antioxid Redox Signal. 2017;27(1):1-20. doi:10.1089/ars.2016.6669

152. Zhang K, Shi Z, Zhang M, et al. Silencing lncRNA Lfar1 alleviates the classical activation and pyoptosis of macrophage in hepatic fibrosis. Cell Death Dis. 2020;11(2):132. doi:10.1038/ s41419-020-2323-5

153. Zhang $\mathrm{T}, \mathrm{Hu} \mathrm{J}$, Wang $\mathrm{X}$, et al. MicroRNA-378 promotes hepatic inflammation and fibrosis via modulation of the NF- $\mathrm{BB}-\mathrm{TNF} \alpha$ pathway. J Hepatol. 2019;70(1):87-96. doi:10.1016/j.jhep.2018.08.026

154. Calvente CJ, Tameda M, Johnson CD, et al. Neutrophils contribute to spontaneous resolution of liver inflammation and fibrosis via microRNA-223. J Clin Invest. 2019;129(10):4091-4109. doi:10.1172/jci122258

155. Chen X, Li HD, Bu FT, et al. Circular RNA circFBXW4 suppresses hepatic fibrosis via targeting the miR-18b-3p/FBXW7 axis. Theranostics. 2020;10(11):4851-4870. doi:10.7150/ thno. 42423 
156. Bala S, Csak T, Saha B, et al. The pro-inflammatory effects of miR-155 promote liver fibrosis and alcohol-induced steatohepatitis. J Hepatol. 2016;64(6):1378-1387. doi:10.1016/j. jhep.2016.01.035

157. Sun NN, Yu CH, Pan MX, et al. Mir-21 mediates the inhibitory effect of Ang (1-7) on AngII-induced NLRP3 inflammasome activation by targeting Spry1 in lung fibroblasts. Sci Rep. 2017;7(1):14369. doi:10.1038/s41598-017-13305-3

158. Zou XZ, Gong ZC, Liu T, et al. Involvement of epithelialmesenchymal transition afforded by activation of LOX-1/ TGF$\beta 1 /$ KLF6 signaling pathway in diabetic pulmonary fibrosis. Pulm Pharmacol Ther. 2017;44:70-77. doi:10.1016/j.pupt.2017.03.012

159. Sun L, Zhu M, Feng W, et al. Exosomal miRNA Let-7 from menstrual blood-derived endometrial stem cells alleviates pulmonary fibrosis through regulating mitochondrial DNA damage. Oxid Med Cell Longev. 2019;2019:4506303. doi:10.1155/2019/ 4506303

160. Li X, Du N, Zhang Q, et al. MicroRNA-30d regulates cardiomyocyte pyroptosis by directly targeting foxo3a in diabetic cardiomyopathy. Cell Death Dis. 2014;5(10):e1479. doi:10.1038/cddis.2014.430

161. Chen A, Chen Z, Xia Y, et al. Liraglutide attenuates NLRP3 inflammasome-dependent pyroptosis via regulating SIRT1/ NOX4/ROS pathway in H9c2 cells. Biochem Biophys Res Commun. 2018;499(2):267-272. doi:10.1016/j.bbrc.2018.03.142

162. Mao Q, Liang XL, Zhang CL, Pang YH, Lu YX. LncRNA KLF3-AS1 in human mesenchymal stem cell-derived exosomes ameliorates pyroptosis of cardiomyocytes and myocardial infarction through miR-138-5p/Sirt1 axis. Stem Cell Res Ther. 2019;10 (1):393. doi:10.1186/s13287-019-1522-4

163. Li F, Zhang L, Xue H, Xuan J, Rong S, Wang K. SIRT1 alleviates hepatic ischemia-reperfusion injury via the miR-182-mediated XBP1/NLRP3 pathway. Mol Ther Nucleic Acids. 2021;23:1066-1077. doi:10.1016/j.omtn.2020.11.015

164. Liang J, Wang Q, Li JQ, Guo T, Yu D. Long non-coding RNA MEG3 promotes cerebral ischemia-reperfusion injury through increasing pyroptosis by targeting miR-485/AIM2 axis. Exp Neurol. 2020;325:113139. doi:10.1016/j.expneurol.2019.113139

165. Cheng J, Liu Q, Hu N, et al. Downregulation of hsa_-

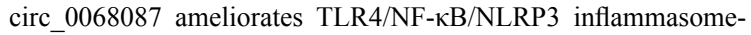
mediated inflammation and endothelial cell dysfunction in high glucose conditioned by sponging miR-197. Gene. 2019;709:1-7. doi:10.1016/j.gene.2019.05.012
166. Han Y, Xu X, Tang C, et al. Reactive oxygen species promote tubular injury in diabetic nephropathy: the role of the mitochondrial ros-txnip-nlrp3 biological axis. Redox Biol. 2018;16:32-46. doi:10.1016/j.redox.2018.02.013

167. Yu J, Nagasu H, Murakami $\mathrm{T}$, et al. Inflammasome activation leads to Caspase-1-dependent mitochondrial damage and block of mitophagy. Proc Natl Acad Sci $U$ S A. 2014;111 (43):15514-15519. doi:10.1073/pnas.1414859111

168. Shi Z, Zhang $\mathrm{K}$, Zhou $\mathrm{H}$, et al. Increased miR-34c mediates synaptic deficits by targeting synaptotagmin 1 through ROSJNK-p53 pathway in Alzheimer's disease. Aging Cell. 2020;19 (3):e13125. doi:10.1111/acel.13125

169. Lin YH. MicroRNA networks modulate oxidative stress in cancer. Int $J$ Mol Sci. 2019;20(18):4497. doi:10.3390/ ijms20184497

170. Zhao XJ, Yu HW, Yang YZ, et al. Polydatin prevents fructose-induced liver inflammation and lipid deposition through increasing miR-200a to regulate Keap1/Nrf2 pathway. Redox Biol. 2018;18:124-137. doi:10.1016/j.redox.2018.07.002

171. Liu J, Yao L, Zhang M, Jiang J, Yang M, Wang Y. Downregulation of LncRNA-XIST inhibited development of non-small cell lung cancer by activating miR-335/SOD2/ROS signal pathway mediated pyroptotic cell death. Aging. 2019;11 (18):7830-7846. doi:10.18632/aging.102291

172. Crane DD, Bauler TJ, Wehrly TD, Bosio CM. Mitochondrial ROS potentiates indirect activation of the AIM2 inflammasome. Front Microbiol. 2014;5:438. doi:10.3389/fmicb.2014.00438

173. $\mathrm{Xu}$ T, Sun L, Shen X, et al. NADPH oxidase 2-mediated NLRP1 inflammasome activation involves in neuronal senescence in hippocampal neurons in vitro. Int Immunopharmacol. 2019;69:60-70. doi:10.1016/j.intimp.2019.01.025

174. Matsui M, Corey DR. Non-coding RNAs as drug targets. Nat Rev Drug Discov. 2017;16(3):167-179. doi:10.1038/nrd.2016.117

175. Chen X, Yang T, Wang W, et al. Circular RNAs in immune responses and immune diseases. Theranostics. 2019;9 (2):588-607. doi:10.7150/thno.29678

176. Piwecka M, Glažar P, Hernandez-Miranda LR, et al. Loss of a mammalian circular RNA locus causes miRNA deregulation and affects brain function. Science. 2017;357:6357. doi:10.1126/ science.aam 8526
Journal of Inflammation Research

\section{Publish your work in this journal}

The Journal of Inflammation Research is an international, peerreviewed open-access journal that welcomes laboratory and clinical findings on the molecular basis, cell biology and pharmacology of inflammation including original research, reviews, symposium reports, hypothesis formation and commentaries on: acute/chronic inflammation; mediators of inflammation; cellular processes; molecular mechanisms; pharmacology and novel anti-inflammatory drugs; clinical conditions involving inflammation. The manuscript management system is completely online and includes a very quick and fair peerreview system. Visit http://www.dovepress.com/testimonials.php to read real quotes from published authors. 